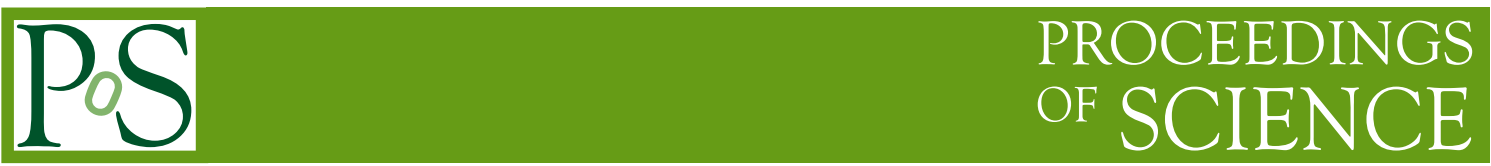

\title{
Overview of the recent CMS Results
}

\author{
Alexander V. Lanyov* (on behalf of the CMS Collaboration) \\ Joint Institute for Nuclear Research (Dubna, Russia) \\ E-mail: Alexander. Lanyovecern.ch
}

We discuss the results of searches for Higgs boson and various new physics phenomena in $p p$ collisions at 7 and $8 \mathrm{TeV}$ delivered by LHC and collected with the CMS detector in 2011-2012. A new boson with a mass near $125 \mathrm{GeV}$ has been observed above the expected background with the most significant excess of events in the two decay modes $\gamma \gamma$ and $Z Z^{*}$. The results of combination of five channels are presented. Limits for new physics phenomena with various experimental signatures (dileptons, diphotons, dijets, multijets, etc.), have been determined. The results of studies of electroweak, top quark and QCD processes and heavy-ion physics are also presented.

XXI International Baldin Seminar on High Energy Physics Problems September 10-15, 2012

JINR, Dubna, Russia

${ }^{*}$ Speaker. 


\section{Introduction}

The Compact Muon Solenoid (CMS) detector has been operating in the proton-proton collisions at the Large Hadron Collider (LHC) since the end of 2009, initially at the center-of-mass energy $\sqrt{s}=7 \mathrm{TeV}$, and then in $2012-$ at $\sqrt{s}=8 \mathrm{TeV}$. The instantaneous luminosity was increasing, reaching $7.5 \cdot 10^{33} \mathrm{~cm}^{-2} \mathrm{~s}^{-1}$ in 2012 , already close to the design value of $10^{34} \mathrm{~cm}^{-2} \mathrm{~s}^{-1}$.

The CMS physics program contains tests of the Standard Model (SM) in various fields (electroweak, top, QCD, etc.) and searches for New physics beyond SM (BSM) predicted by different models [1]. A special attention is paid to the only missing fundamental particle in SM - the Higgs boson, solving the mystery of introducing finite masses of the fundamental elementary particles in a renormalizable way $[2,3]$. There is also a program of studies of heavy-ion physics in lead-lead and proton-lead collisions at the unprecedented energy leading to more distinctive signals of hot and dense medium production.

An integrated recorded luminosity around $22 \mathrm{fb}^{-1}$ was obtained in the 2012 run and around $5.5 \mathrm{fb}^{-1}$ in the 2011 run [4], due to the excellent performance of LHC and CMS. This enables CMS to study the electroweak physics and search for new physics with large statistics (using millions of $Z$ and $W$ vector bosons, and an opportunity to explore the regions of high invariant dilepton masses up to 2-3 TeV), top physics (using thousands of $t \bar{t}$ pairs, studying also the single top production at several percent level), effect of jets up to $p_{T}^{\text {jet }}=2 \mathrm{TeV}$, dijets up to $m_{\text {jet }}=5 \mathrm{TeV}$.

In the search for Higgs boson, several channels reached the sensitivity of the SM, including also high-resolution channels $H \rightarrow \gamma \gamma$ and $H \rightarrow Z Z^{*} \rightarrow 4 \ell$. The hot results were reported on July 4 , 2012 at a dedicated scientific seminar at CERN by CMS and ATLAS collaborations, presenting the evidence of a new boson with a mass around $125 \mathrm{GeV}[5,6]$.

In this report, section 2 briefly introduces the CMS detector, section 3 is devoted to SM physics, section 4 describes the results of Higgs boson searches, section 5 - searches for new physics, section 6 - heavy ion physics, conclusions are given at the end.

\section{CMS Detector}

The (CMS Compact Muon Solenoid) detector is one of the two general purpose detectors located at the LHC (see Fig. 1). The central feature of the CMS detector is a superconducting solenoid providing an axial magnetic field of $3.8 \mathrm{~T}$ and enclosing the all-silicon inner tracker, the crystal electromagnetic calorimeter (ECAL), and the brass-scintillator hadronic calorimeter (HCAL). The inner tracker is composed of a pixel detector and a silicon strip tracker, and measures charged-particle trajectories in the pseudorapidity range $|\eta|<2.5$. The finely segmented ECAL consists of nearly 76000 lead-tungstate crystals which provide coverage in pseudorapidity up to $|\eta|=3.0$. The muon system covers the pseudorapidity region $|\eta|<2.4$ and consists of up to four stations of gas-ionization muon detectors installed outside the solenoid and sandwiched between the layers of the steel return yoke. More details on the CMS detector and its performance can be found in papers $[7,8]$. 


\section{Standard Model}

The measurements of electroweak, top, QCD, etc. processes are necessary for testing SM itself and making its phenomenological aspects more precise (e.g. parton distribution functions) enabling more accurate predictions.

\subsection{Electroweak physics}

One of the first studies in the field of the electroweak physics is the measurement of the inclusive $W$ and $Z$ boson cross sections. This study was started in 2010 at $\sqrt{s}=7 \mathrm{TeV}$ using both electron and muon final states [9], in 2012 first results for $\sqrt{s}=8 \mathrm{TeV}$ were reported in [10] for the integrated luminosity of $18.7 \pm 0.9 \mathrm{pb}^{-1}$ yielding the measured inclusive cross sections of $\sigma(\mathrm{pp} \rightarrow \mathrm{WX}) \times \mathrm{Br}(\mathrm{W} \rightarrow \ell v)=11.88 \pm 0.03$ (stat.) \pm 0.22 (syst.) \pm 0.52 (lumi.) nb and $\sigma(\mathrm{pp} \rightarrow \mathrm{ZX}) \times \operatorname{Br}\left(\mathrm{Z} \rightarrow \ell^{+} \ell^{-}\right)=1.12 \pm 0.01$ (stat.) \pm 0.02 (syst.) \pm 0.05 (lumi.) nb, limited to a dilepton mass range of 60 to $120 \mathrm{GeV}$. The measured values are consistent within the electron and muon channels, and agree with the next-to-next-to-leading order (NNLO) QCD calculations $[11,12]$.

Other studies of electroweak physics at CMS included measurements of differential cross sections of $Z / \gamma^{*}$ production on transverse momentum $p_{T}$, rapidity, as well as the differential Drell-Yan cross section $d \sigma / d M$ in the dimuon and dielectron channels shown in Fig. 2, and the double-differential cross section $d^{2} \sigma / d M d Y$ in the dimuon channel, using the full 2011 dataset at $\sqrt{s}=7 \mathrm{TeV}[13,14]$. In general, the results are in a good agreement with the NNLO theoretical predictions which are required for a correct description of the $d \sigma / d M$ results at low dimuon

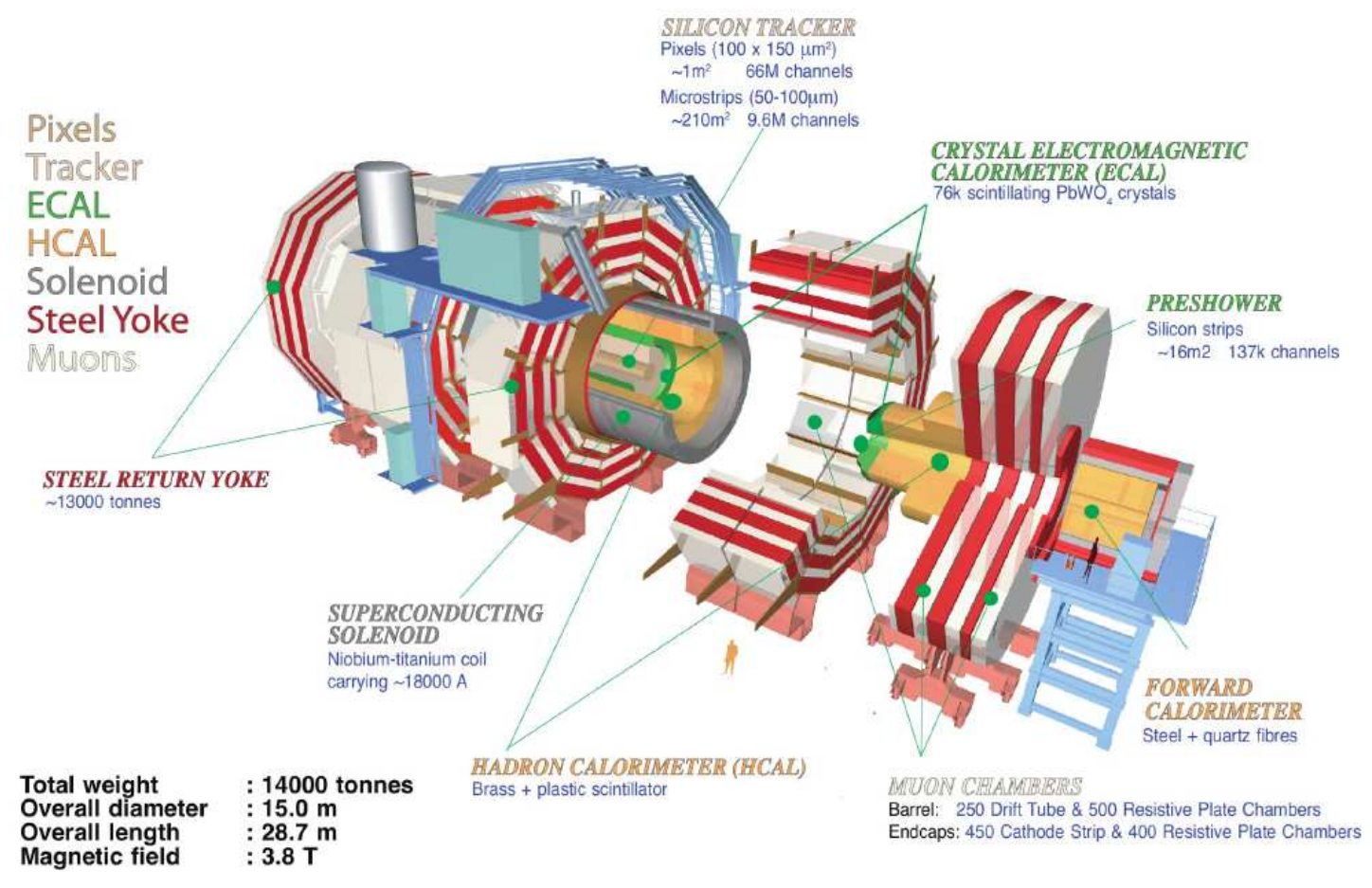

Figure 1: CMS detector scheme. 

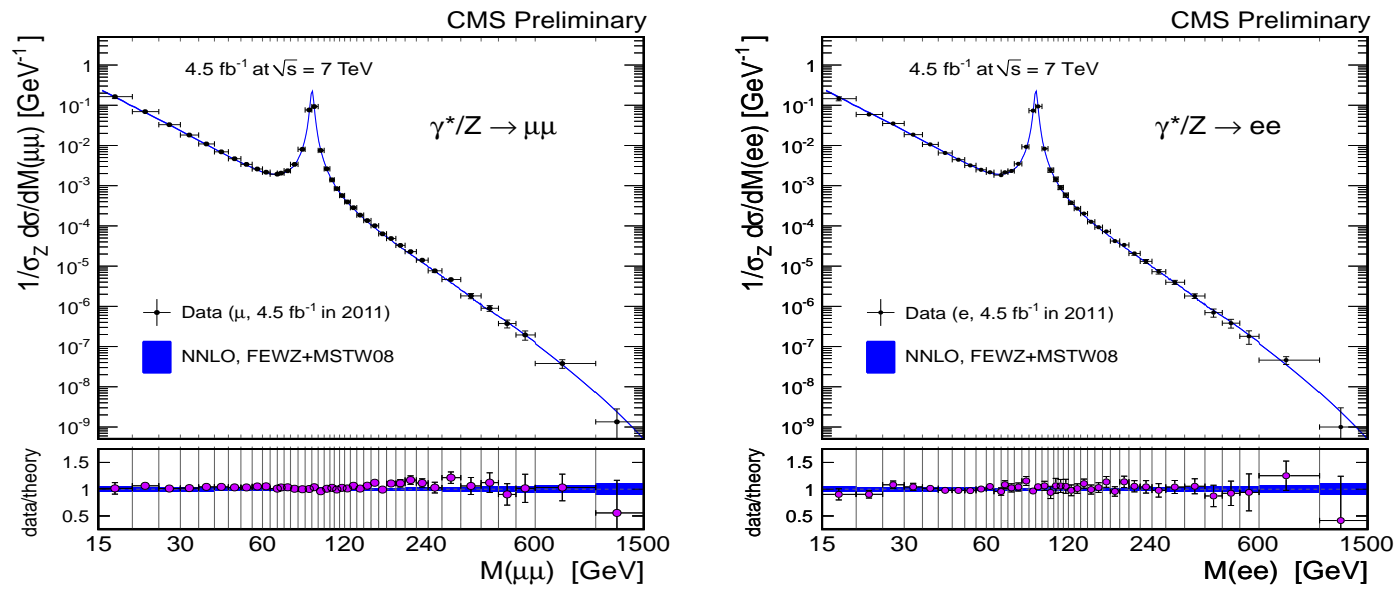

Figure 2: The Drell-Yan invariant mass spectrum, normalized to the $Z$ resonance region, $r=$ $\left(1 / \sigma_{l l} d \sigma / d M\right)$, as measured by CMS and predicted by NNLO calculations, for the full phase space. The vertical error bar indicates the experimental (statistical and systematic) uncertainties summed in quadrature with the theory uncertainty resulting from the model-dependent kinematic distributions inside each bin in the dimuon (left) and the dielectron channels (right).

invariant masses, also extending our knowledge of the partonic contents of the proton. Forwardbackward asymmetry of Drell-Yan pair production and Weinberg weak mixing angle $\sin _{W}^{2}$ have been measured to be consistent with SM model predictions [15,16]. Diboson production cross section has been measured in $W W$ [17] and $Z Z$ [18] channels at $\sqrt{s}=8 \mathrm{TeV}$, and at $\sqrt{s}=7 \mathrm{TeV}$ [19].

The general summary of the main electroweak physics measurements by CMS is shown in Fig. 3 using the results published in $[9,10,17-24]$. No deviations of the measured values from SM predictions have been found.

\subsection{Top quark physics}

CMS recently presented the results for the top quark mass with a dataset up to $5 \mathrm{fb}^{-1}$ at $\sqrt{s}=$ $7 \mathrm{TeV} m_{t}=173.36 \pm 0.38$ (stat) \pm 0.91 (syst) $\mathrm{GeV}$, using the combination of lepton+jets, dilepton and all-jets channels, the result of the $\mu+$ jet channel dominates [25].

The cross section of $t \bar{t}$ production was measured by CMS at $\sqrt{s}=7 \mathrm{TeV}$ to be $\sigma_{t \bar{t}}=165.8 \pm$ 2.2 (stat) \pm 10.6 (syst) \pm 7.8 (lumi) pb [26] in a good agreement with the SM prediction of $\sigma_{t \bar{t}}=$ $167_{-18}^{+17} \mathrm{pb}$ [27]. Since $t \bar{t}$ cross section is related to the strong coupling constant $\alpha_{s}$ with approximate NNLO QCD predictions, this allowed to determine $\alpha_{s}\left(M_{Z}\right)=0.1178_{-0.0040}^{+0.0046}$ from $t \bar{t}$ events at CMS [28]. This value of $\alpha_{s}$ is in a good agreement with the current world average, its precision is similar to the most precise hadron collider measurements. For this analysis top quark mass value from the world average $m_{t}=173.2 \pm 1.4 \mathrm{GeV}$ was used. Measurements at $\sqrt{s}=8 \mathrm{TeV}$ with $2.4 \mathrm{fb}^{-1}$ produced the value $\sigma_{t \bar{t}}=227 \pm 3$ (stat) \pm 11 (syst) \pm 10 (lumi) pb [29] in a good agreement with the SM prediction of $\sigma_{t \bar{t}}=225.2 \pm 0.11 \mathrm{pb}$ at NLO.

Single top production at a hadron collider is described by several types of Feynman diagrams shown in Fig. 4, the cross sections have been measured by CMS for different channels: $t$-channel $-\sigma_{t}=80.1 \pm 5.7 \pm 11.0 \pm 4.0 \mathrm{pb}$ using $5.0 \mathrm{fb}^{-1}$ at $8 \mathrm{TeV}$ [30], and at $7 \mathrm{TeV}: t$-channel $\sigma_{t}=67.2 \pm 6.1 \mathrm{pb}$ using $1.17-1.56 \mathrm{fb}^{-1}$ [31], $t W$-channel $-\sigma_{t W}=16_{-4}^{+5} \mathrm{pb}$ using $4.9 \mathrm{fb}^{-1}$ [32]. 


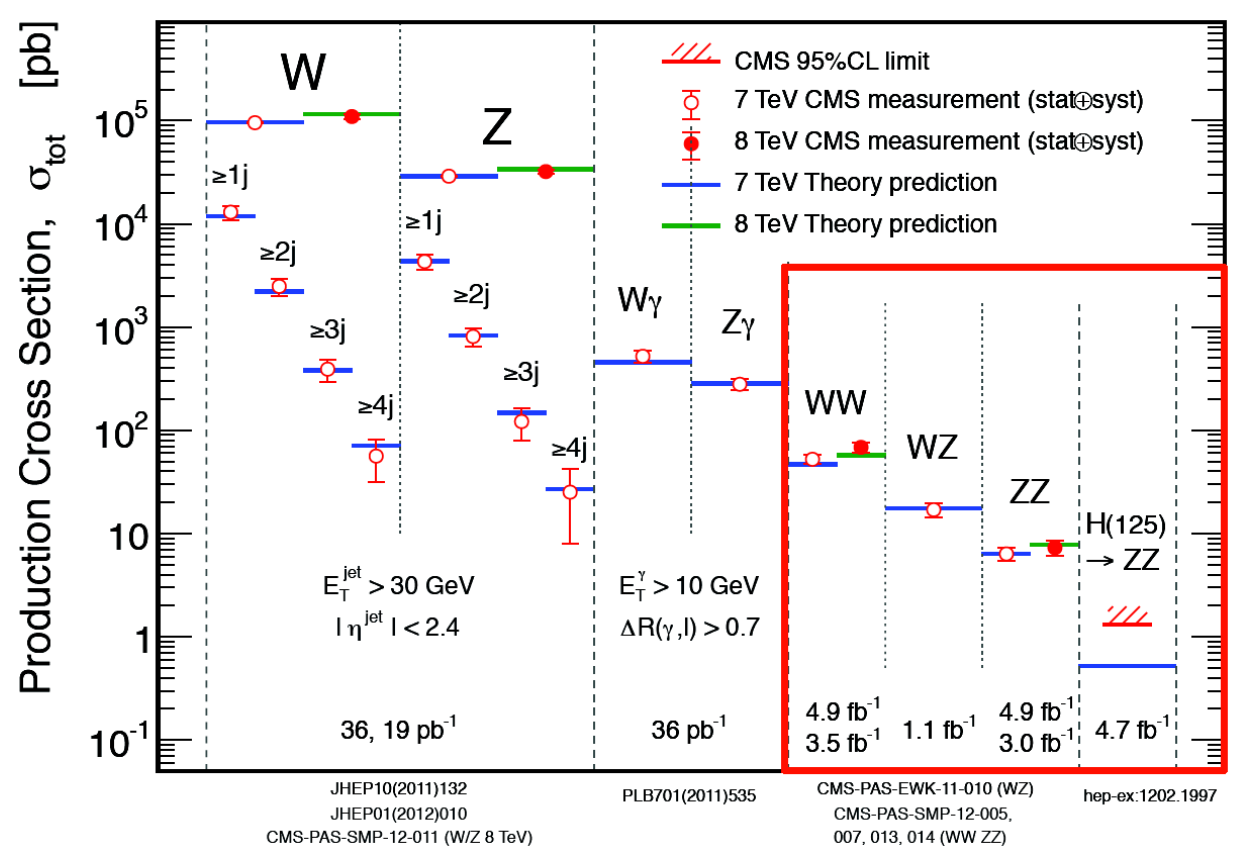

Figure 3: Summary of the main electroweak physics results at CMS.

The work is also going on measuring $s$-channel where the theoretical prediction is the smallest one: $\sigma_{s}^{\mathrm{SM}, 7 \mathrm{TeV}}=4.63 \pm 0.07_{-0.17}^{0.18} \mathrm{pb}$ [33], for other channels the measurements agree with the theoretical predictions $\sigma_{t}^{\mathrm{SM}, 8 \mathrm{TeV}}=87.2_{-0.7-1.7}^{+2.1+1.5} \mathrm{pb}[34], \sigma_{t}^{\mathrm{SM}, 7 \mathrm{TeV}}=64.6_{-2.8}^{+2.7} \mathrm{pb}[35], \sigma_{t W}^{\mathrm{SM}, 7 \mathrm{TeV}}=$ $15.6 \pm 0.4_{-1.2}^{+1.0} \mathrm{pb}[36]$. CMS has also measured the differential cross section of the top quark production on the mass, rapidity and transverse momentum of the top-antitop system [37], as well as it has performed the first measurement of the cross section of the associative production of $t \bar{t}$ pair with vector bosons [38].
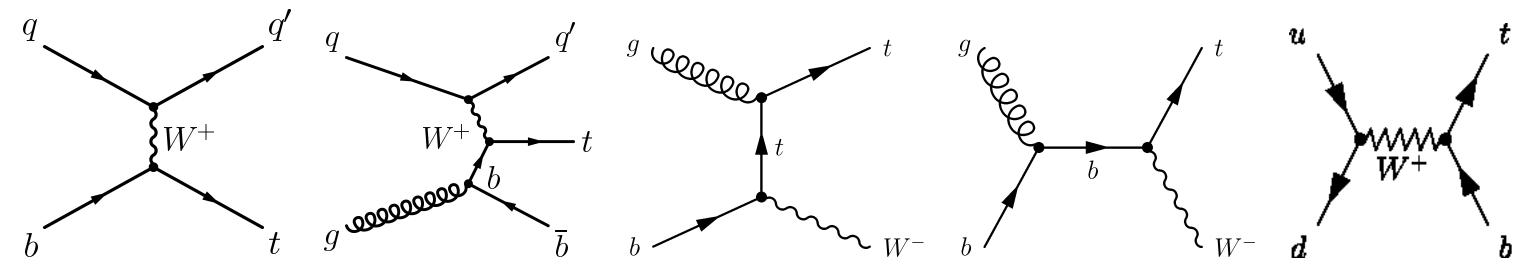

Figure 4: Leading order Feynman diagrams for single top production in the $t$-channel (the first and second plots), $t W$-channel (the third and fourth plots), and $s$-channel (the fifth plot).

The top and anti-top quarks are unpolarized in $t \bar{t}$ pair production - the top quark polarization was measured to be $P_{n}=-0.009 \pm 0.029 \pm 0.041$ [39] in a good agreement with SM. However, the quarks in $t \bar{t}$ pairs are correlated due to the production process. Spin correlations can be measured from a fit to the angular distribution between leptons in the dilepton events, the resulting helicity asymmetry $A_{\text {Helicity }}=0.24 \pm 0.02$ (stat) \pm 0.08 (syst) [40] is compatible with SM prediction $A_{\text {Helicity }}^{\mathrm{SM}}=0.31$.

\subsection{QCD physics}

At the start of 2009 and 2010 runs the multiplicities of charged hadrons have been measured 

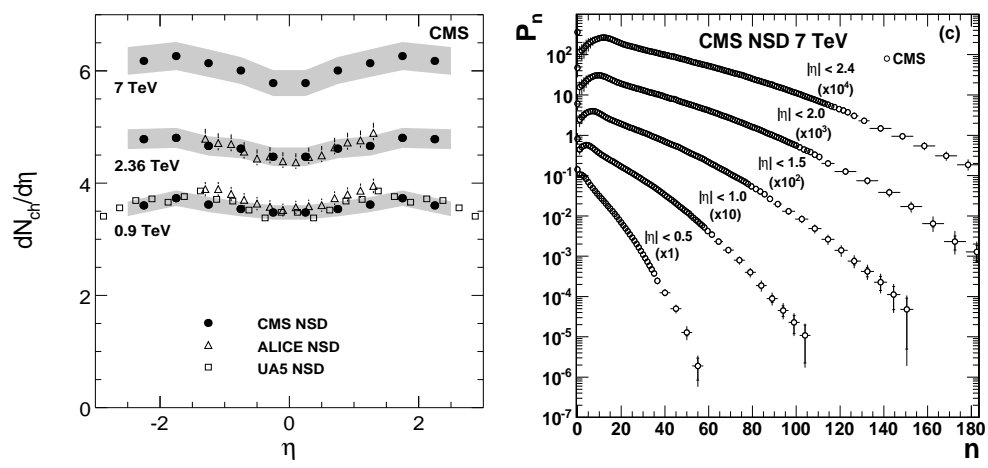

Figure 5: Multiplicities of charged hadrons measured by CMS at $\sqrt{s}=7 \mathrm{TeV}$.
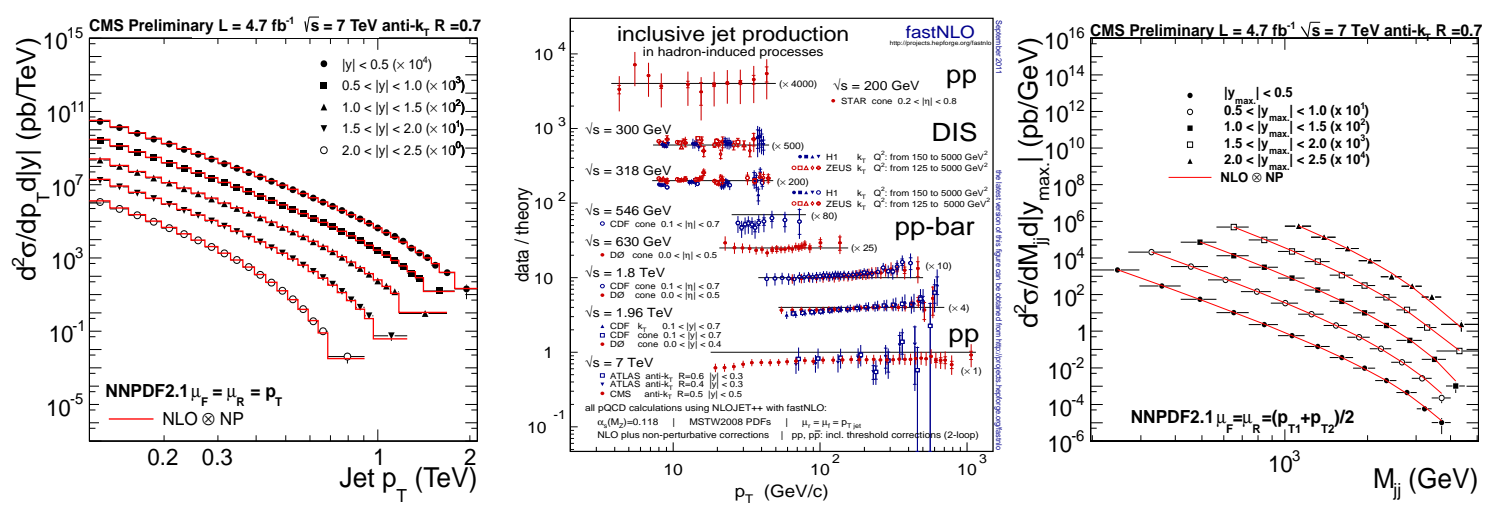

Figure 6: Double differential cross section of inclusive jets (the left plot) and dijets (the right plot), the fit of experimental data by different experiments (the middle plot).

at $\sqrt{s}=0.9,2.36,7 \mathrm{TeV}[41,42]$, see Fig. 5. Studies of jets included measurement of the inclusive jet cross sections [43] giving results in a good agreement with NLO QCD until $p_{T}^{\text {jet }} \approx 2 \mathrm{TeV}$, see Fig. 6 (left plot). The results are used in general fits of collider data by fastNLO collaboration [44], see Fig. 6 (middle plot). Dijet studies included measurement of differential dijet cross sections up to invariant dijet mass of $5 \mathrm{TeV}$ [43] (see Fig. 6 the right plot), angular distributions allowed to put limits for quark compositeness $\Lambda^{ \pm}=5.6-6.7 \mathrm{TeV}$ [45]. Results for the ratio of the inclusive 3 -jet to 2-jet cross sections are in a good agreement with SM for all values of the total transverse momentum $H_{T}$ between 0.5 and $2.5 \mathrm{TeV}$, extending to the $H_{\mathrm{T}}$ range that has not been explored before [46].

QCD event shapes provide information about the properties of hadronic final states, sensitive to $\mathrm{QCD}$ radiation and gluon emission changing the shape of the energy flow. In the CMS analysis [47], the event-shape variables were used in analogy to the ones applied at lepton colliders [48, 49]. Dijet azimuthal decorrelations were also studied [50] comparing the differential dijet cross section vs the azimuthal separation $\Delta \phi$ between the two leading jets with the pQCD predictions at leading and next-to-leading orders, as well as predictions of various Monte Carlo generators. These measurements provide input to improve currently available models of QCD multijet production. 


\section{Search for Higgs boson}

The Standard Model is based on the mechanism of spontaneous symmetry breaking by introducing a scalar field with a non-zero mean value, leading to existence of a Higgs boson [51-56]. In the beginning of 2000s' the LEP experiments excluded Higgs masses less than $114.4 \mathrm{GeV}$ [57]. The fit of the precision electroweak measurements gave a prediction of $m_{H}=99_{-23}^{+28} \mathrm{GeV}$ [58]. Using the 2011 data collected at the LHC the CMS excluded the Higgs boson mass range 127.5$600 \mathrm{GeV}$ and gave an evidence of the peak at $125 \mathrm{GeV}$ having a significance of 2.8 standard deviation $(\sigma)[59,60]$. In 2012 the Fermilab experiments reported an excess of their combined data inconsistent with the background prediction at the level around $3 \sigma$ [61].

On July 4, 2012, a dedicated seminar was held in CERN where the CMS and ATLAS collaborations (each) reported $\approx 5 \sigma$ observation of a new boson with a mass about $125 \mathrm{GeV}$ consistent with the SM Higgs boson. The following section describes the results reported by the CMS collaboration in the subsequent publication in the Physics Letters journal [5].

The search for Higgs boson was performed in five channels with the largest expected significance at the LHC: $\gamma \gamma, Z Z^{*} \rightarrow 4 \ell, W W \rightarrow l v l v, \tau \tau, b \bar{b}$, the main characteristics of them can be seen in Table 1. The first two channels provide the best significance and separation from the background due to a good mass resolution of $1-2 \%$, the other channels have the largest production cross sections. The used data are up to $5.1 \mathrm{fb}^{-1}$ at $\sqrt{s}=7 \mathrm{TeV}$ and $5.3 \mathrm{fb}^{-1}$ at $\sqrt{s}=8 \mathrm{TeV}$.

\begin{tabular}{|c|c|c|c|c|c|}
\hline \multirow{2}{*}{$\begin{array}{l}\text { Decay } \\
\text { mode }\end{array}$} & \multirow{2}{*}{$\begin{array}{c}\text { Production } \\
\text { tagging }\end{array}$} & \multirow{2}{*}{$\begin{array}{c}\text { No. of } \\
\text { subchannels }\end{array}$} & \multirow{2}{*}{$\begin{array}{c}m_{H} \text { range } \\
(\mathrm{GeV})\end{array}$} & \multicolumn{2}{|c|}{ Int. Lum. $\left(\mathrm{fb}^{-1}\right)$} \\
\hline & & & & $7 \mathrm{TeV}$ & $8 \mathrm{TeV}$ \\
\hline$\gamma \gamma$ & $\begin{array}{c}\text { untagged } \\
\text { dijet (VBF) }\end{array}$ & $\begin{array}{c}4 \\
1 \text { or } 2\end{array}$ & $110-150$ & 5.1 & 5.3 \\
\hline$Z Z^{*}$ & untagged & 3 & $110-600$ & 5.1 & 5.3 \\
\hline$W W$ & $\begin{array}{c}\text { untagged } \\
\text { dijet (VBF) }\end{array}$ & $\begin{array}{c}4 \\
1 \text { or } 2\end{array}$ & $110-600$ & 4.9 & 5.1 \\
\hline$\tau \tau$ & $\begin{array}{c}\text { untagged } \\
\text { dijet (VBF) }\end{array}$ & $\begin{array}{c}16 \\
4\end{array}$ & $110-145$ & 4.9 & 5.1 \\
\hline $\mathrm{bb}$ & lepton, $E_{T}^{\text {miss }}(\mathrm{VH})$ & 10 & $110-135$ & 5.0 & 5.1 \\
\hline
\end{tabular}

Table 1: Summary of the subchannels, or categories, used in the analysis of each decay mode of Higgs boson. (VBF is vector boson fusion, $\mathrm{VH}$ is associated vector production).

The search for $\gamma \gamma$ has used multivariate techniques to select and classify the events [62,63]. As an independent cross-check, the analysis has also been performed that is almost identical to the one described in the 2011 analysis paper [63], using simpler criteria based on the properties of the reconstructed photons to select and classify events. The multivariate analysis has achieved the $15 \%$ higher sensitivity than the cross-check analysis. The weighted histogram of the mass spectrum is shown in Fig. 7 (top left), the resulting $p$-value, a probability for a background fluctuation to be at least as large as the observed maximum excess, is shown in Fig. 7 (top right), corresponding to a significance of $4.1 \sigma$ at $125 \mathrm{GeV}$. The signal strength, defined as the production cross section times the relevant branching ratios relative to the SM expectation, is $\mu=\sigma / \sigma_{\mathrm{SM}}=1.6 \pm 0.4$. 

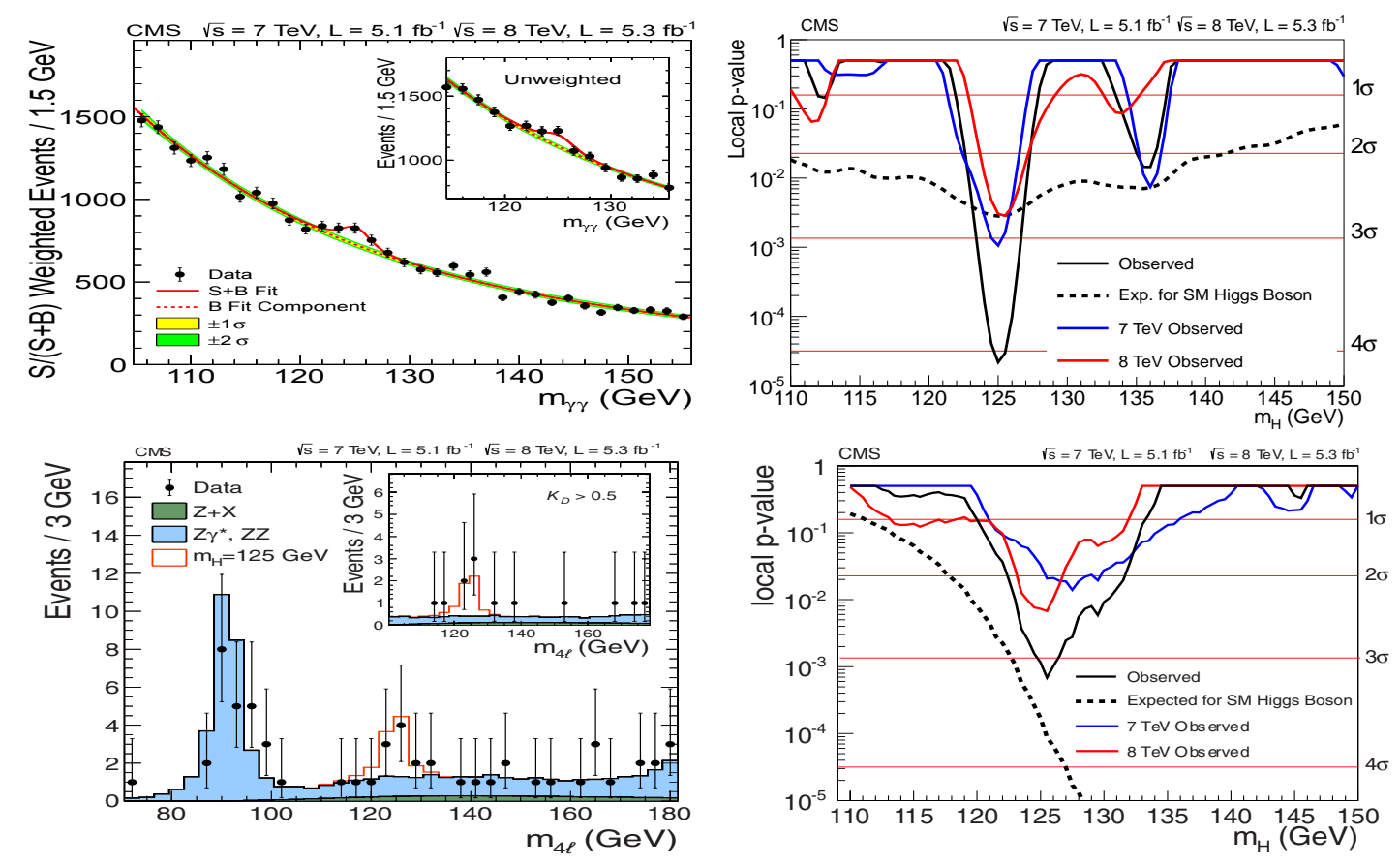

Figure 7: The $\gamma \gamma$ (the top plots) and $Z Z^{*}$ channels (the bottom plots) in the search for the Higgs boson.

The search in $Z Z^{*}$ channels used three possible subchannels of dilepton decays of $Z$ boson: $4 \mu, 2 \mu 2 e$ and $4 e$. This is a clean channel where two high-mass pairs of opposite-sign isolated muons or electrons coming from the primary vertex were required. The main backgrounds included irreducible $Z Z$ production [18] and reducible backgrounds: $Z+$ jets, $Z b \bar{b}, t \bar{t}, W Z$ production. The resulting mass spectrum can be seen in Fig. 7 (bottom left) and the numerical statistics for the channel can be seen in Table 2. The SM branching ratio of $H \rightarrow Z Z^{*}$ is of the order of $10^{-3}$ at $125 \mathrm{GeV}$ [64], therefore a specialized method utilizing the difference of matrix elements for the SM Higgs boson and the background was used to enhance the analysis sensitivity: Matrix Element Likelihood Approach (MELA) [65, 66]. Using all available five angles of four leptons (left plot in Fig. 8) and the masses of both $Z$ candidates, $M_{Z_{1}}$ and $M_{Z_{2}}$, one can construct a kinematic discriminant $K_{D}=P_{\mathrm{Sig}} /\left(P_{\mathrm{Sig}}+P_{\mathrm{Bkg}}\right)$ based on the probability ratio of the signal and background hypotheses

\begin{tabular}{|l|c|c|c||c|}
\hline Channel & $4 e$ & $4 \mu$ & $2 e 2 \mu$ & $4 \ell$ \\
\hline \hline$Z Z$ background & $2.7 \pm 0.3$ & $5.7 \pm 0.6$ & $7.2 \pm 0.8$ & $15.6 \pm 1.4$ \\
$Z+X$ & $1.2_{-0.8}^{+1.1}$ & $0.9_{-0.6}^{+0.7}$ & $2.3_{-1.4}^{+1.8}$ & $4.4_{-1.7}^{+2.2}$ \\
\hline All backgrounds $\left(110<m_{4 \ell}<160 \mathrm{GeV}\right)$ & $4.0 \pm 1.0$ & $6.6 \pm 0.9$ & $9.7 \pm 1.8$ & $20 \pm 3$ \\
\hline Observed $\left(110<m_{4 \ell}<160 \mathrm{GeV}\right)$ & 6 & 6 & 9 & 21 \\
\hline \hline Signal $\left(m_{H}=125 \mathrm{GeV}\right)$ & $1.36 \pm 0.22$ & $2.74 \pm 0.32$ & $3.44 \pm 0.44$ & $7.54 \pm 0.78$ \\
\hline \hline All backgrounds (signal region) & $0.7 \pm 0.2$ & $1.3 \pm 0.1$ & $1.9 \pm 0.3$ & $3.8 \pm 0.5$ \\
\hline Observed (signal region) & 1 & 3 & 5 & 9 \\
\hline
\end{tabular}

Table 2: Statistics of $H \rightarrow Z Z^{*} \rightarrow 4 \ell$ subchannels. 

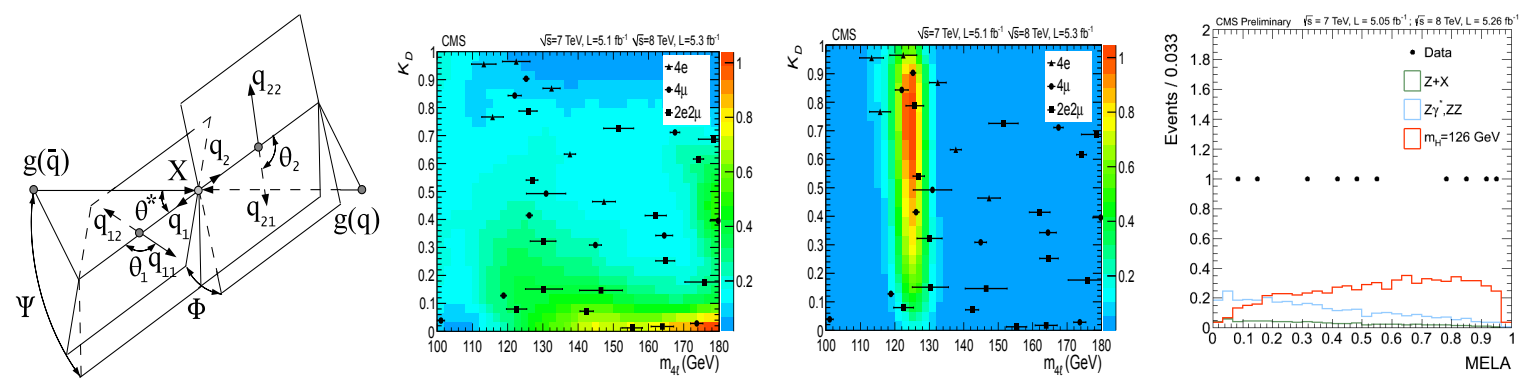

Figure 8: Scheme of available angles of four lepton system (left plot); Distribution of events $Z Z^{*} \rightarrow 4 \ell$ (filled symbols defined in the legend) for the kinematic discriminant $K_{D}$ versus $m_{4 l}$, the color-coded regions show the event density expected from the background (the second plot) and a SM Higgs boson $\left(m_{H}=125 \mathrm{GeV}\right.$, the third plot). The events are marked by filled symbols (defined in the legend), the horizontal error bars indicate the estimated mass resolution; The distribution of the kinematic discriminant $K_{D}$ for the background and a SM Higgs boson is shown in the rightmost plot.

to increase the expected significance by $15-20 \%$. The second and third plots of Fig. 8 show the 2D distributions for the expected background and SM Higgs signal, respectively; one can see that the experimental points are well described by the SM Higgs signal hypothesis, especially for the large values of MELA discriminant $K_{D}$. The resulting plot for $p$-value is shown in Fig. 7 (bottom right) corresponding to a significance of $3.1 \sigma$. The resulting signal strength is $\mu=\sigma / \sigma_{\mathrm{SM}}=0.7_{-0.3}^{+0.4}$.

The $95 \%$ confidence level (C.L.) limits as a function of mass for the other three channels are given in Fig. 9. The combination of all five channels yielded a local significance of $5.0 \sigma$, see Table 3 and Fig. 10. The signal strength (ratio of the fitted cross section of the excess near $125 \mathrm{GeV}$ to $\mathrm{SM}$ cross section) is consistent with the SM scalar boson expectation: $\sigma / \sigma_{\mathrm{SM}}=$ $0.87 \pm 0.23$. The signal strengths in 7 and $8 \mathrm{TeV}$ data are consistent. Fig. 11 shows the signal strengths per channels, the combined signal strength as a function of Higgs mass, and 2D plot of signal strength vs mass. The measurement of the mass of the new boson yielded value of $M_{X}=125.3 \pm 0.4$ (stat.) \pm 0.5 (syst.) $\mathrm{GeV}$, which is dominated by the $\gamma \gamma$ channel.

\begin{tabular}{|l|c|c|}
\hline Decay mode/combination & Expected $(\sigma)$ & Observed $(\sigma)$ \\
\hline \hline$\gamma \gamma$ & 2.8 & 4.1 \\
$Z Z^{*}$ & 3.6 & 3.1 \\
\hline$\tau \tau+b b$ & 2.4 & 0.4 \\
$\gamma \gamma+Z Z^{*}$ & 4.7 & 5.0 \\
$\gamma \gamma+Z Z^{*}+W W$ & 5.2 & 5.1 \\
$\gamma \gamma+Z Z^{*}+W W+\tau \tau+b b$ & 5.8 & 5.0 \\
\hline
\end{tabular}

Table 3: Expected and observed significance values of the SM Higgs boson search for different channels and their combinations.

First measurements of the scaling of vector vs fermion couplings of the new boson $c_{\mathrm{V}} \approx 1$, $c_{\mathrm{F}} \approx 0.5$ are consistent with the SM Higgs boson hypothesis. The detailed study of the boson couplings will continue as the data are accumulated in the future [67]. 

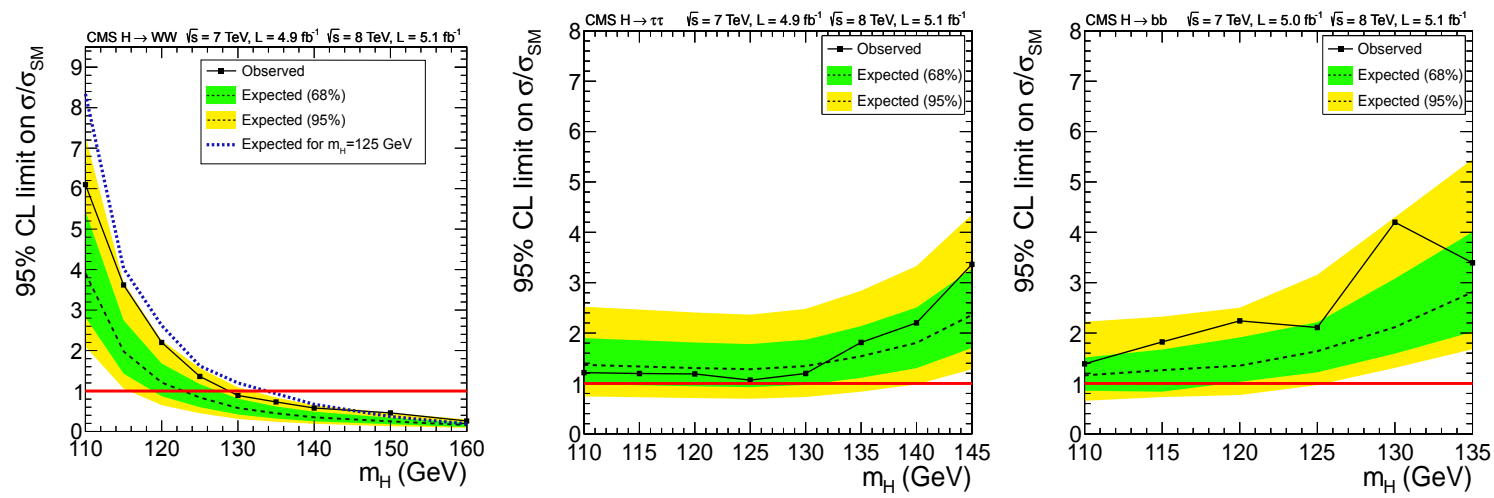

Figure 9: $95 \%$ C.L. limits for $W W, \tau \tau, b \bar{b}$ channels in the search for the SM Higgs boson.
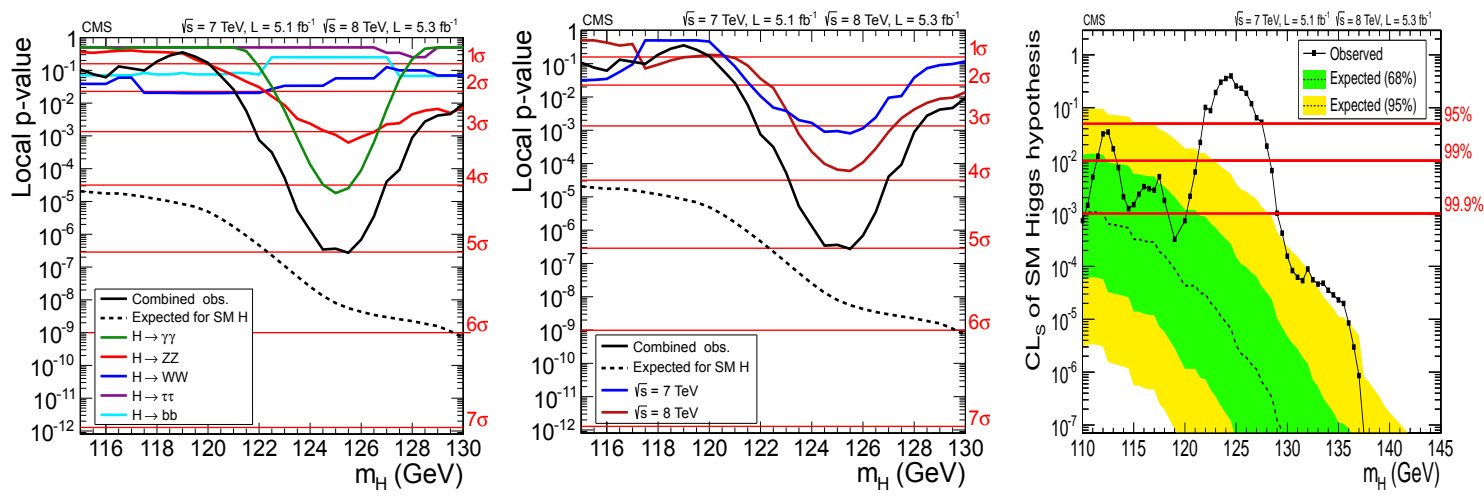

Figure 10: Combination of all channels for Higgs boson search.
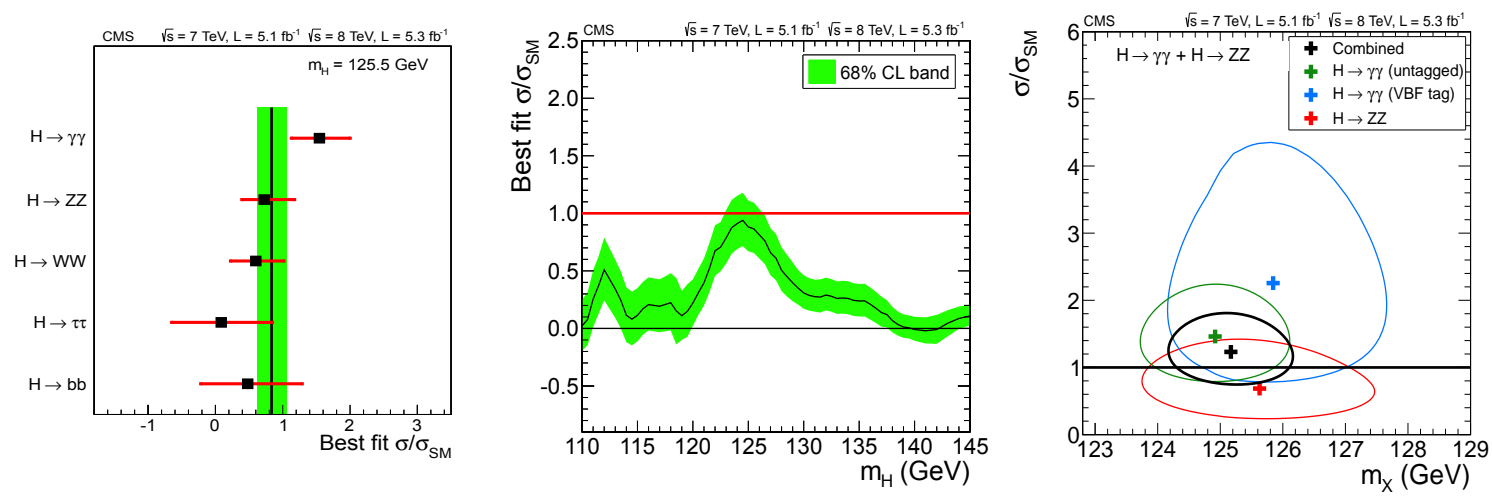

Figure 11: Signal strengths per channels, combined signal strength as a function of Higgs mass, and 2D plot of signal strength vs mass. 
The analysis presented in [5] is based on the statistics of $10.4 \mathrm{fb}^{-1}$ which was insufficient to determine the $C P$ and spin properties of the new boson (SM requires $J^{P C}=0^{++}$state). It is only known that spin 1 is excluded due to Landau-Yang theorem $[68,69] .{ }^{1}$ The projections based on Monte Carlo simulation $[66,70]$ have indicated that $25-30 \mathrm{fb}^{-1}$ data expected to be collected by the end of 2012 data taking at CMS (including the data collected in 2011) could yield measurements of these properties. ${ }^{2}$ A new era starts to measure the new boson properties, including also spin and parity determination. The collection of future data will enable a more rigorous test of the properties of the new boson and an investigation whether the properties of the new particle imply physics beyond SM.

\section{Search for New Physics}

The search for new physics at CMS is performed in various channels for different theoretical models predicting deviations from SM. One of the important directions is the search for narrow resonances, in particular, in the dilepton channels (the dimuon and dielectron ones). Many models of new physics predict the existence of narrow resonances at the TeV mass scale decaying to a pair of charged leptons [73, 74] and exotic hadron states [75], in particular, Sequential Standard Model $Z_{\text {SSM }}^{\prime}$ with SM-like couplings, the $Z_{\psi}^{\prime}$ predicted by grand unified theories [73], and KaluzaKlein graviton excitations arising in the Randall-Sundrum (RS) model of possible warped extra dimension scenario with one extra spatial dimension [76,77]. The recent measurement by CMS used the data at $\sqrt{s}=8 \mathrm{TeV}$ and integrated luminosities up to $4.1 \mathrm{fb}^{-1}$ in both the dimuon and dielectron channels $[78,79]$. The previous best direct limits on the $Z_{\mathrm{SSM}}^{\prime}$ and $Z_{\psi}^{\prime}$ masses obtained at $\sqrt{s}=7 \mathrm{TeV}$ were $2330 \mathrm{GeV}$ and $2000 \mathrm{GeV}$, respectively [80]. The search for resonances is based on a shape analysis of dilepton mass spectra in order to be robust against uncertainties in the absolute background level. The spectra are consistent with expectations from SM and the upper limits have been determined on the product of the cross section and branching fraction for $Z^{\prime}$ into lepton pairs relative to the $\mathrm{SM} Z$ boson production. The obtained upper limits on the cross section ratio

$$
R_{\sigma}=\frac{\sigma\left(p p \rightarrow Z^{\prime}+X \rightarrow \ell \ell+X\right)}{\sigma(p p \rightarrow Z+X \rightarrow \ell \ell+X)}
$$

at 95\% C.L. are shown in Fig. 12 (top plots) both for the dilepton channels and their combination. The following $95 \%$ C.L. lower limits on the mass of $Z^{\prime}$ resonances are obtained using the combinations of data obtained at $\sqrt{s}=7$ and $8 \mathrm{TeV}: 2590 \mathrm{GeV}$ for $Z_{\mathrm{SSM}}^{\prime}$ and $2270 \mathrm{GeV}$ for $Z_{\psi}^{\prime}$. RS Kaluza-Klein gravitons are excluded below 2390 (2030) $\mathrm{GeV}$ for coupling $k / \bar{M}_{\mathrm{Pl}}=0.10(0.05)$, where $k$ is the curvature of the extra dimension and $\bar{M}_{\mathrm{Pl}}$ is the reduced effective Planck scale. With new LHC data CMS will be able to extend the mass range for these analyses well beyond the present highest points.

\footnotetext{
${ }^{1}$ However, hypothetically signal in $\gamma \gamma$ channel could arise from $H \rightarrow a a$, where pseudoscalar state $a$ having a mass of the order of tens of $\mathrm{MeV}$ could decay to $\gamma \gamma$, so the photons could be highly collimated and may be identified as a single photon in the electromagnetic calorimeter [71].

${ }^{2}$ Please see the CMS talk at HCP'2012 conference in November 2012 for a recent update for the parity measurement of the new boson [72].
} 

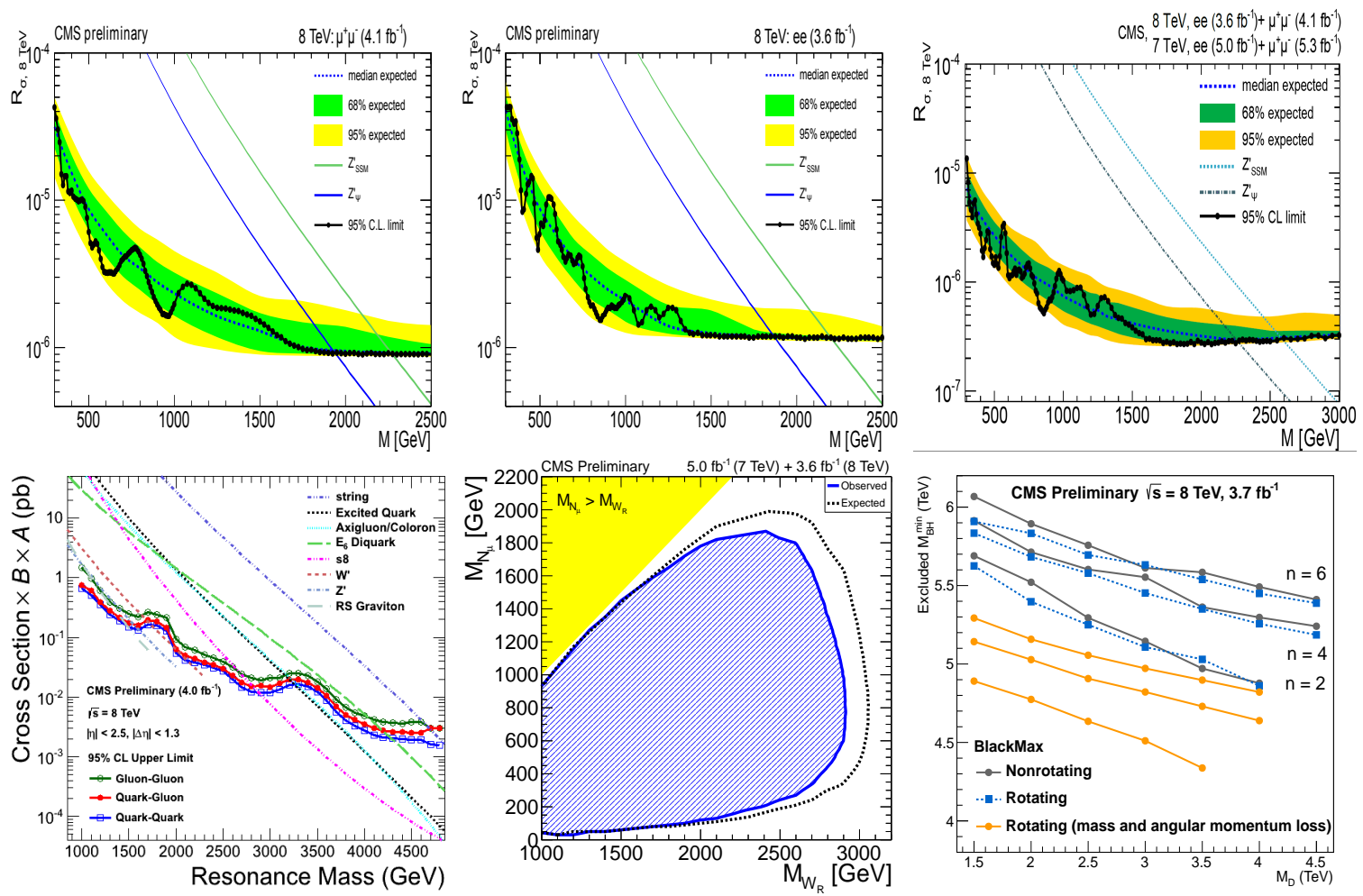

Figure 12: Obtained limits in the search for new physics: In the top row - 95\% C.L. limits for the product of $Z^{\prime}$ cross section and braching ratios in different channels: $\mu^{+} \mu^{-}, e^{+} e^{-}$, and combined $l^{+} l^{-}$, from left to right. In the bottom row: limits for masses of narrow dijet resonances, heavy neutrino and right-handed $W_{R}$ boson, and minimum masses of black holes as a function of the reduced Planck scale.

Similarly, CMS performed searches in other $Z^{\prime}$ channels: $\tau$-lepton pair $\tau \tau$ [81], dibosons $Z Z$ [82], $t \bar{t}$ pair [83], anomalous production of highly boosted $Z$ bosons decaying to dimuons [63]; and $W^{\prime}$ channels: semileptonic $l v$ [84], diboson $W Z$ [85] and heavy quarks $b t$ [86].

Besides, narrow resonances in the dijet channel have been studied and the upper limits at the $95 \%$ C.L. on the resonance cross section have been determined (Fig. 12 bottom left plot). By comparing these generic limits with theoretical predictions for the cross section of several models of new particles, CMS sets specific lower limits on the mass of string resonances, excited quarks, axigluons, colorons, s8 resonances, $\mathrm{E}_{6}$ diquarks, $W^{\prime}$ and $Z^{\prime}$ bosons, and $\mathrm{RS}$ gravitons in the $1-4.7 \mathrm{TeV}$ range, many of which extend previous exclusions from the dijet mass search technique [87-89].

CMS has performed a search for signals from the production of right-handed $W_{R}$ bosons and heavy neutrinos $N_{\ell}(\ell=e, \mu)$, that arise naturally in the left-right symmetric extension to SM [90], no excess over expectations from SM processes was observed [91]. For models with an exact leftright symmetry, and assuming that either $N_{e}$ or $N_{\mu}$ is the only right-handed neutrino accessible at LHC energies, CMS excluded the region in the two-dimensional parameter $\left(M_{W_{R}}, M_{N_{\ell}}\right)$ space that extends beyond $M_{W_{R}}=2.5 \mathrm{TeV}$. Assuming degenerate neutrino masses for all neutrino flavors, and combining the $8 \mathrm{TeV}$ electron and muon channel results, exclusion in the $\left(M_{W_{R}}, M_{N_{\ell}}\right)$ mass plane extending to $M_{W_{R}}=2.8 \mathrm{TeV}$ was obtained (Fig. 12 bottom middle plot). Combining the 7 and $8 \mathrm{TeV}$ data for the muon channel only, and assuming that $N_{\mu}$ is light enough to be produced at the 
LHC, CMS excluded right-handed $W_{R}$ boson up to mass $M_{W_{R}}=2.9 \mathrm{TeV}$.

One of the most spectacular predictions of theories with the low-scale quantum gravity is an opportunity of microscopic black hole production in proton-proton collisions at the LHC energies $[92,93]$. Such models are motivated mainly by the puzzling large difference between the electroweak scale $(\sim 0.1 \mathrm{TeV})$ and the Planck scale $\left(M_{\mathrm{Pl}} \sim 10^{16} \mathrm{TeV}\right)$, known as the hierarchy problem. CMS has released a new analysis at $\sqrt{s}=8 \mathrm{TeV}$ with $3.7 \mathrm{fb}^{-1}$ [94] to search for the black hole production in a model with $n$ large, flat, extra spatial dimensions (ADD model) [95,96]. Events with the large total transverse energy have been analyzed for the presence of multiple energetic jets, leptons, and photons, which are typical signals of evaporating semiclassical and quantum black holes, and string balls. Agreement with the expected SM backgrounds, which are dominated by QCD multijet production, has been observed for various combined multiplicities of jets and other reconstructed objects in the final state. Model-independent limits have been determined on new physics processes producing high-multiplicity, energetic final states. In addition, new model-specific indicative limits have been also found excluding semiclassical black holes with masses below 4.1 to $6.1 \mathrm{TeV}$, see Fig. 12 (bottom right) for the minimum black hole mass excluded at 95\% C.L. as function of the reduced Planck scale for various BlackMax black hole models without the stable remnant and a number of extra dimensions of $n=2,4,6$ [97]. The analysis benefits substantially from the increased sensitivity at the higher collision energy compared to the searches at $\sqrt{s}=7 \mathrm{TeV}$ published previously by CMS $[98,99]$. The results of the analysis could be also suitable for other models in which heavy objects appear and decay to final states with a large scalar sum of objects' transverse energies of the order of several $\mathrm{TeV}$.

Many other searches for deviations from SM were carried out in CMS, see Fig. 13 for the graphical summary [100]. CMS also performed searches for supersymmetry producing improved limits for cross sections and masses. Fig. 14 (the left plot) displays the best exclusion limits for the masses of the primary supersymmetric particles from several CMS supersymmetry searches at $\sqrt{s}=7 \mathrm{TeV}$ in a Simplified Model Spectra framework, for different topologies defined in [101]. Two scenarios are used: a small mass $\approx 0 \mathrm{GeV}$ of the lightest supersymmetric particle (LSP), as well as the fixed splitting of $200 \mathrm{GeV}$ between the primary particles and LSP. Fig. 14 (the right plot) shows the observed limits from several CMS supersymmetry searches at $\sqrt{s}=7 \mathrm{TeV}$ in the Constrained Minimal Supersymmetric Standard Model (CMSSM) plotted in $\left(m_{0}, m_{1 / 2}\right)$ plane, where $m_{0}$ is the common mass of all scalar particles and $m_{1 / 2}$ - the common gaugino mass [102].

\section{Heavy ion physics}

CMS has a vast research program in the heavy ion physics [103]. The data with $\mathrm{PbPb}$ collisions were obtained at the nucleon-nucleon center-of-mass energy $\sqrt{s_{N N}}=2.76 \mathrm{TeV}$ with the integrated luminosity of 10 and $155 \mu \mathrm{b}^{-1}$ in 2010 and 2011, respectively. The very start of the data taking has shown a strong typical imbalance in the transverse momentum of the leading and subleading jets in the central $\mathrm{PbPb}$ collisions [104], see the left plot in Fig. 15. This is consistent with a high degree of jet quenching in the produced matter. A large fraction of the momentum balance of these unbalanced jets is carried by low- $p_{T}$ particles at a large radial distance. The results provide qualitative constraints on the nature of the jet modification in $\mathrm{PbPb}$ collisions and quantitative input to models of the transport properties of the medium produced in these collisions. 


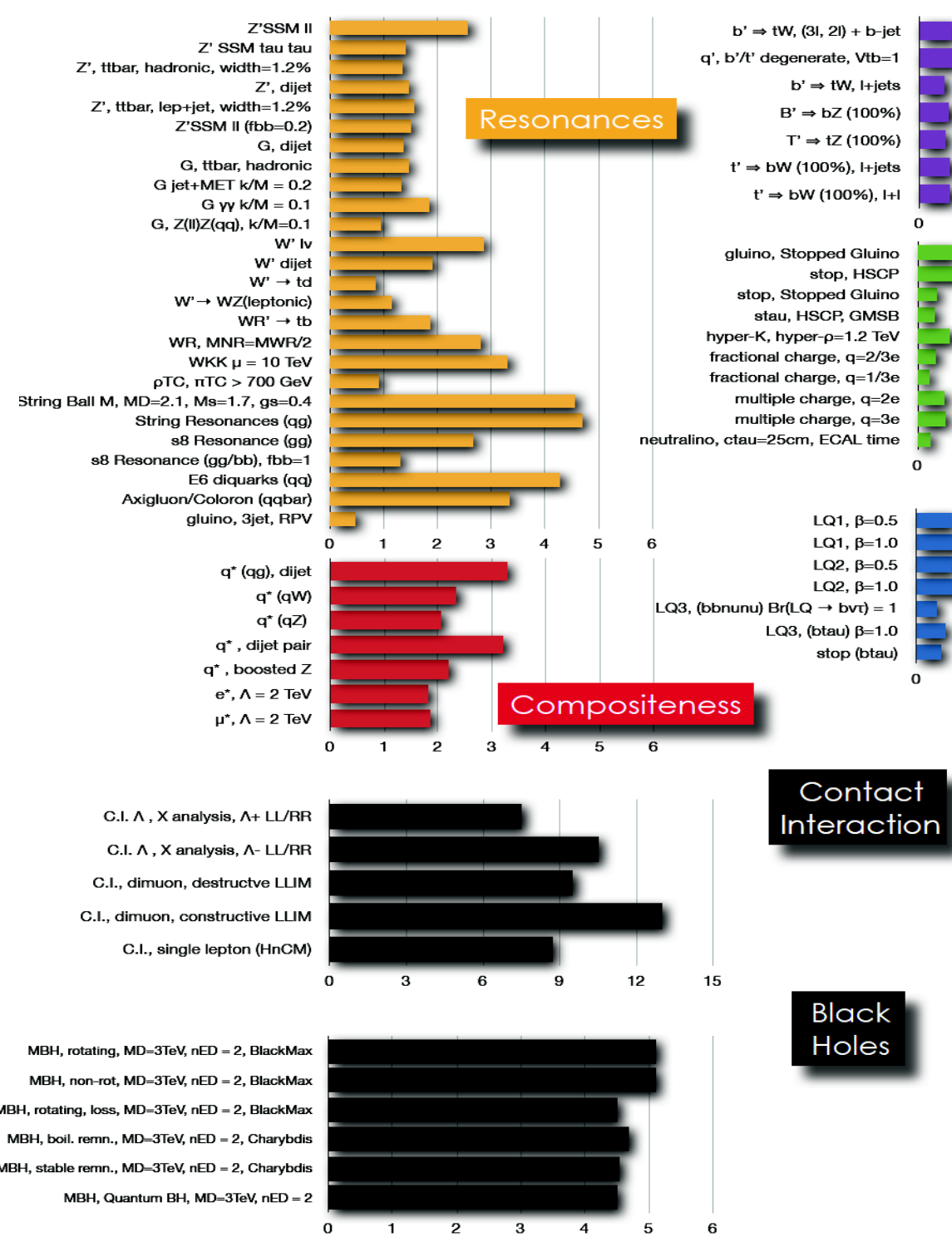

Figure 13: Summary of $95 \%$ C.L. limits for masses in the New Physics physics searches at CMS.
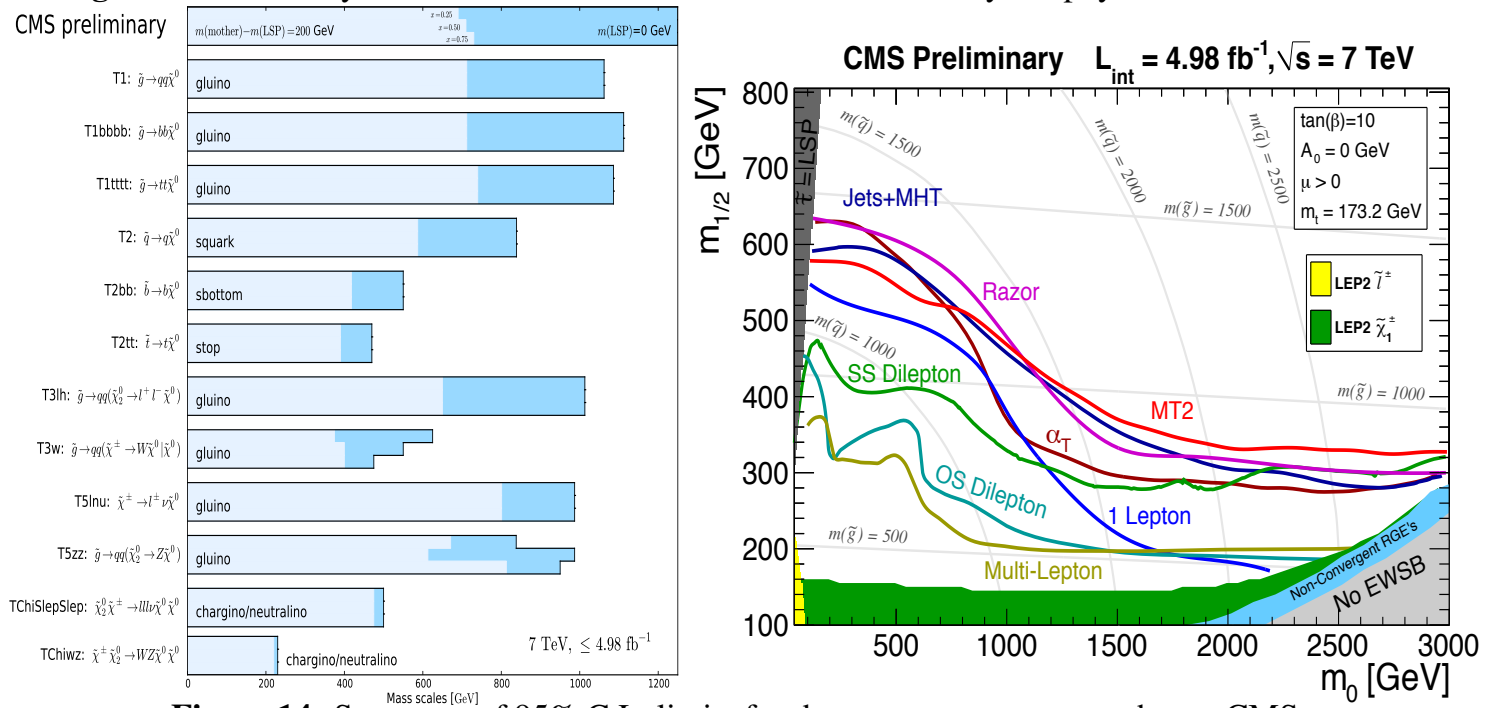

Figure 14: Summary of $95 \%$ C.L. limits for the supersymmetry searches at CMS. 

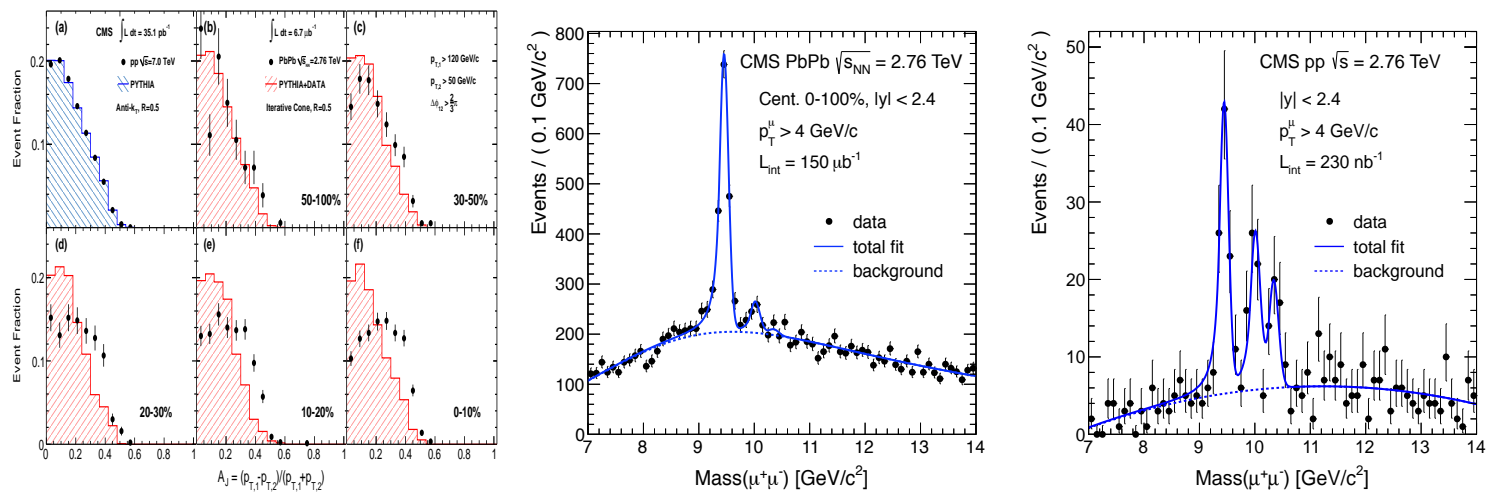

Figure 15: Characteristic distributions for heavy ion collisions. Left plots: distribution of $p_{T}$ imbalance for leading / subleading jets for increasing centrality. Other plots: the dimuon mass spectrum in the region of $\Upsilon(1 S-3 S)$ peaks in $\mathrm{PbPb}$ and $p p$ collisions.
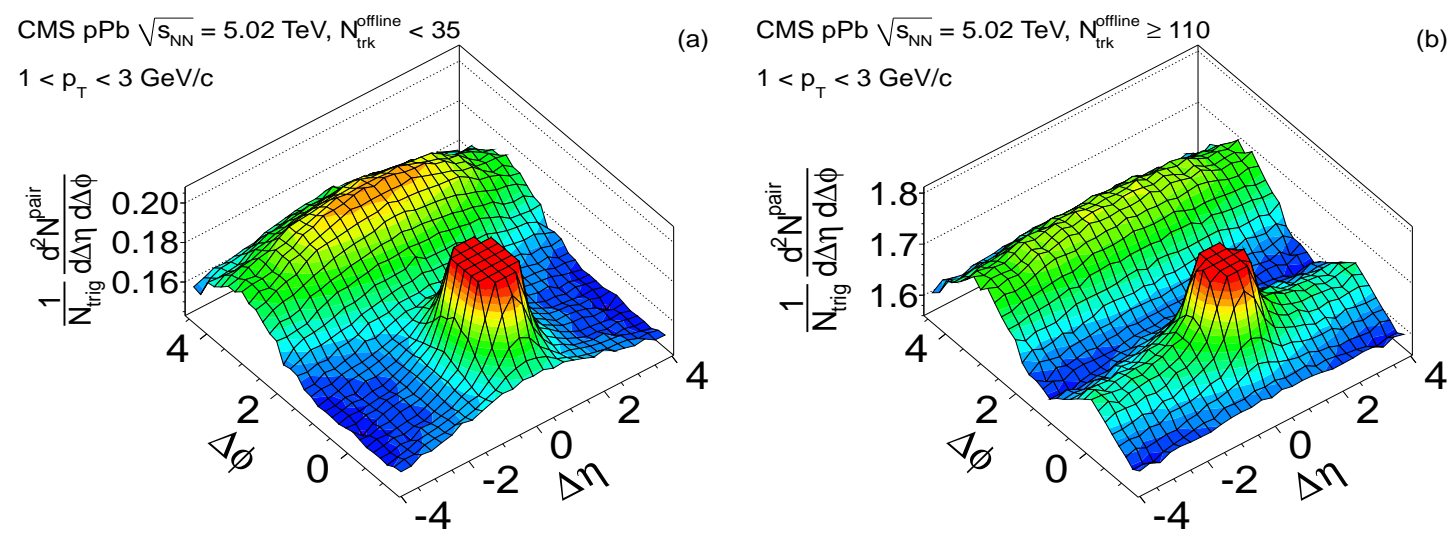

(c) CMS $N \geq 110, p_{T}>0.1 \mathrm{GeV} / \mathrm{c}$

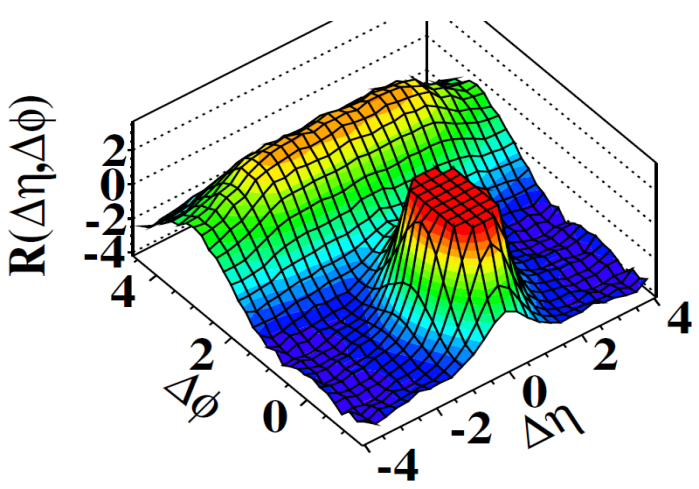

(d) CMS N $\geq 110,1.0 \mathrm{GeV} / \mathrm{c}<\mathrm{p}_{\mathrm{T}}<3.0 \mathrm{GeV} / \mathrm{c}$

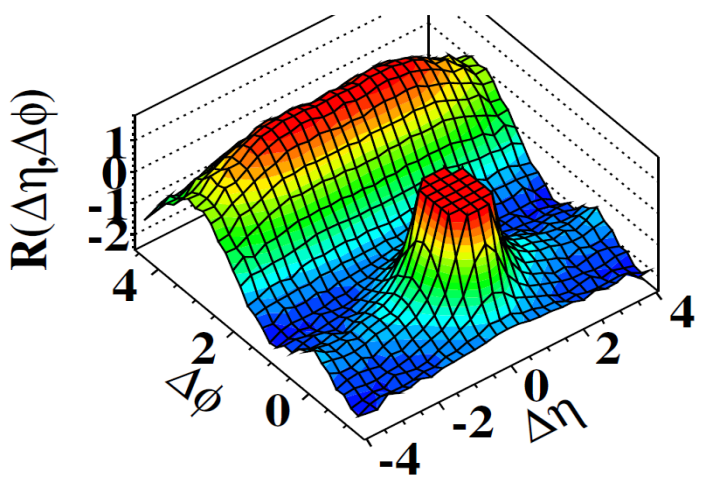

Figure 16: Top plots: 2-D two-particle correlation functions for $\sqrt{s_{N N}}=5.02 \mathrm{TeV} \mathrm{pPb}$ collisions for pairs of charged particles with $1<p_{T}<3 \mathrm{GeV}$. Results are shown (a) for low-multiplicity and (b) high-multiplicity selection. The sharp near-side peaks from jet correlations have been truncated to better illustrate the structure outside that region. Bottom plots: similar plots for the "ridge" effect in $p p$ collisions for $\sqrt{s}=7 \mathrm{TeV}$. 
In September 2012 a short run of proton-lead collisions was performed at the LHC with the proton-nucleon center-of-mass energy of $\sqrt{s_{N N}}=5.02 \mathrm{TeV}$ and the integrated luminosity of $1 \mu \mathrm{b}$. In high-multiplicity events, a long-range $(2<|\Delta \eta|<4)$, near-side $(\Delta \phi \approx 0)$ structure emerges in the two-particle $\Delta_{\eta}-\Delta \phi$ correlation functions [105], see Fig. 16 (top plots). This is the first observation of such correlations in proton-nucleus collisions, resembling the ridge-like correlations seen in high-multiplicity $p p$ collisions at $\sqrt{s}=7 \mathrm{TeV}$ [106] (Fig. 16 bottom plots) and in heavy ion collisions over a broad range of center-of-mass energies [107]. The correlation demonstrates a pronounced maximum in the range of $p_{T}=1-1.5 \mathrm{GeV}$ and an approximately linear increase with the charged particle multiplicity for high-multiplicity events. The observations at $\mathrm{pp}$ and $\mathrm{pPb}$ collisions are qualitatively similar when selecting the same observed particle multiplicity, while the overall strength of the correlations is significantly larger in $\mathrm{pPb}$ collisions.

The heavy-ion results also included an observation of sequential suppression of higher Upsilon resonances $\Upsilon(2 S), \Upsilon(3 S)[108,109]$ compared to $p p$ collisions (see last two plots in Fig. 15), the study of high- $p_{T}$ charged particle suppression compared to $p p$ collisions [110], suppression of non-prompt $J / \psi$, prompt $J / \psi$, and $Y(1 S)$ [111], $Z$ boson production [112].

\section{Conclusions}

Excellent performance of LHC and CMS has provided a large dataset of $p p$ collisions, $5 \mathrm{fb}^{-1}$ at $\sqrt{s}=7 \mathrm{TeV}$ and more than $20 \mathrm{fb}^{-1}$ at $\sqrt{s}=8 \mathrm{TeV}$. In the search for the SM Higgs boson, a new boson with a mass around $125 \mathrm{GeV}$ has been found in the analysis of several channels. Its properties are compatible with the properties of the SM Higgs boson within the present experimental uncertainties. The studies of the processes in the Standard Model and searches for new physics beyond the Standard Model have been performed. The electroweak results on $Z / \gamma^{*}$ and $W$ differential distributions on the mass, transverse momentum, rapidity are used for parton distribution functions and studies of theoretical higher-order calculations. In the search for new physics the experiment has managed, in particular, to exclude new particles in the 2-3 TeV range in the dilepton channels and $5 \mathrm{TeV}$ for dijets, allowing to go beyond the previous studies. By now, CMS has published around 200 papers in scientific journals [113], this number continues to grow.

Further efforts should increase the precision of measurements and give the answers for some burning questions from the theory side: whether supersymmetry exists or it can eventually be ruled out for the energies of LHC, whether extra dimensions can be found and the branching ratios of the rare decays correspond to the Standard Model predictions, etc. The analysis of the whole dataset taken in 2012, and the planned transition to the full design LHC center-of-mass energy of 13$14 \mathrm{TeV}$ can provide answers to, at least, some of these questions, and there is also a chance to discover new unexplained features of high-energy physics, not yet predicted by the theory, as we have already seen in the past.

\section{Acknowledgments}

The author would like to express the gratitude to the members of CMS Collaboration for fruitful cooperation. 


\section{References}

[1] CMS Collaboration, CMS Technical Design Report, volume II: Physics performance, J. Phys. G 34 (2007) 995, doi:10.1088/0954-3899/34/6/S01.

[2] L. B. Okun, Particle physics prospects: August 1981, in Proc. of the 1981 Intern. Symp. on Lepton and Photon Interactions at High Energy, 24-29 August, 1981, Bonn, Germany, Ed. W. Pfeil. Bonn: Bonn Univ., Phys. Inst., 1981, p. 1018; http://lss.fnal.gov/conf/C810824/p1018.pdf

[3] V. A. Rubakov, On Large Hadron Collider's discovery of a new particle with Higgs boson properties, Uspekhi Fiz. Nauk, 182 (2012) No.10 1017, doi:10.3367/UFNr.0182.201210a.1017.

[4] CMS Collaboration, Public CMS Luminosity Information web page https://twiki.cern.ch/twiki/bin/view/CMSPublic/LumiPublicResults

[5] CMS Collaboration, Observation of a new boson at a mass of $125 \mathrm{GeV}$ with the CMS experiment at the LHC, Phys. Lett. B 716 (2012) 30 [arXiv:1207.7235], doi:10.1016/j.physletb.2012.08.021.

[6] ATLAS Collaboration, Observation of a new particle in the search for the Standard Model Higgs boson with the ATLAS detector at the LHC, Phys. Lett. B 716 (2012) 1 [arXiv:1207.7214], doi:10.1016/j.physletb.2012.08.020.

[7] CMS Collaboration, CMS Technical Design Report, volume I: Detector Performance and Software, CERN-LHCC-2006-001.

[8] CMS Collaboration, The CMS experiment at the CERN LHC, JINST 3 (2008) S08004, doi:10.1088/1748-0221/3/08/S08004.

[9] CMS Collaboration, Measurement of the Inclusive $W$ and $Z$ Production Cross Sections in pp Collisions at $\sqrt{s}=7 \mathrm{TeV}$, JHEP 1110 (2011) 132 [arXiv:1107.4789].

[10] CMS Collaboration, Inclusive W/Z cross section at 8 TeV, CMS-PAS-SMP-12-011.

[11] K. Melnikov and F. Petriello, The W boson production cross section at the LHC through $O\left(\alpha_{s}^{2}\right)$, Phys. Rev. Lett. 96 (2006) 231803 [arXiv:hep-ph/0603182].

[12] A. D. Martin, W. J. Stirling, R. S. Thorne and G. Watt, Parton distributions for the LHC, Eur. Phys. J. C 63 (2009) 189 [arXiv:0901.0002].

[13] CMS Collaboration, Measurement of the differential and double-differential Drell-Yan cross section in proton-proton collisions at $7 \mathrm{TeV}$, CMS-PAS-EWK-11-007.

[14] CMS Collaboration, Measurement of the Drell-Yan Cross Section in pp Collisions at $\sqrt{s}=7 \mathrm{TeV}$, JHEP 1110 (2011) 007 [1108.0566].

[15] CMS Collaboration, Forward-backward asymmetry of Drell-Yan lepton pairs in pp collisions at $\sqrt{s}=7$ TeV, Phys. Lett. B 718 (2013) 699 [arXiv:1207.3973].

[16] CMS Collaboration, Measurement of the weak mixing angle with the Drell-Yan process in proton-proton collisions at the LHC, Phys. Rev. D 84 (2011) 112002 [arXiv:1110.2682].

[17] CMS Collaboration, Measurement of WW production rate, CMS-PAS-SMP-12-013.

[18] CMS Collaboration, Measurement of $Z Z$ production cross section in $Z Z \rightarrow 2 l 2 l^{\prime}$ decay channel in pp collisions at $\sqrt{s}=8 \mathrm{TeV}$, CMS-PAS-SMP-12-014.

[19] CMS Collaboration, Measurement of the WW, WZ and ZZ cross sections at CMS, CMS-PAS-EWK-11-010. 
[20] CMS Collaboration, Observation of Z decays to four leptons with the CMS detector at the LHC, JHEP 1212 (2012) 034 [arXiv:1210.3844].

[21] CMS Collaboration, Jet Production Rates in Association with $W$ and $Z$ Bosons in pp Collisions at $\sqrt{s}=7 \mathrm{TeV}$, JHEP 1201 (2012) 010 [arXiv:1110.3226].

[22] CMS Collaboration, Measurement of $W \gamma$ and $Z \gamma$ production in pp collisions at $\sqrt{s}=7$ TeV, Phys. Lett. B 701 (2011) 535 [arXiv:1105.2758].

[23] CMS Collaboration, Measurement of WW production rate, CMS-PAS-SMP-12-005.

[24] CMS Collaboration, Search for the standard model Higgs boson in the decay channel $H \rightarrow Z Z \rightarrow 4 \ell$ in pp collisions at $\sqrt{s}=7$ TeV, Phys. Rev. Lett. 108 (2012) 111804 [arXiv:1202.1997].

[25] CMS Collaboration, Top mass combination, CMS-PAS-TOP-11-018.

[26] CMS Collaboration, Combination of ATLAS and CMS top-quark pair cross section measurements using proton-proton collisions at $\sqrt{s}=7 \mathrm{TeV}$, CMS-PAS-TOP-12-003, ATLAS-CONF-2012-166.

[27] M. Aliev, H. Lacker, U. Langenfeld et al., HATHOR: HAdronic Top and Heavy quarks crOss section calculatoR, Comput. Phys. Commun. 182 (2011) 1034 [arXiv:1007.1327].

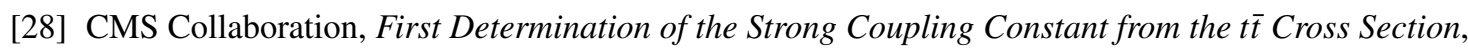
CMS-PAS-TOP-12-022.

[29] CMS Collaboration, Top pair cross section in dileptons, CMS-PAS-TOP-12-007.

[30] CMS Collaboration, Measurement of the single-top t-channel cross section in pp collisions at centre-of-mass energy of $8 \mathrm{TeV}$, CMS-PAS-TOP-12-011.

[31] CMS Collaboration, Measurement of the single-top-quark t-channel cross section in pp collisions at $\sqrt{s}=7 \mathrm{TeV}$, arXiv:1209.4533.

[32] CMS Collaboration, Evidence for associated production of a single top quark and $W$ boson in pp collisions at $\sqrt{s}=7 \mathrm{TeV}$, [arXiv:1209.3489].

[33] N. Kidonakis, NNLL resummation for s-channel single top quark production, Phys. Rev. D 81 (2010) 054028 [arXiv:1001.5034].

[34] N. Kidonakis, Differential and total cross sections for top pair and single top production, arXiv:1205.3453.

[35] N. Kidonakis, Next-to-next-to-leading-order collinear and soft gluon corrections for t-channel single top quark production, Phys. Rev. D 83 (2011) 091503 [arXiv:1103.2792].

[36] N. Kidonakis, Two-loop soft anomalous dimensions for single top quark associated production with $a W^{-}$or $H^{-}$, Phys. Rev. D 82 (2010) 054018 [arXiv:1005.4451].

[37] CMS Collaboration, Measurement of differential top-quark pair production cross sections in pp collisions at $\sqrt{s}=7 \mathrm{TeV}$, arXiv:1211.2220.

[38] CMS Collaboration, Measurement of the Associated Production of Vector Bosons with Top-Antitop Pairs at $7 \mathrm{TeV}$, CMS-PAS-TOP-12-014.

[39] CMS Collaboration, Measurement of the top polarization in the dilepton final state, CMS-PAS-TOP-12-016.

[40] CMS Collaboration, Measurement of Spin Correlations in t̄̄ production, CMS-PAS-TOP-12-004. 
[41] CMS Collaboration, Transverse-momentum and pseudorapidity distributions of charged hadrons in pp collisions at $\sqrt{s}=7$ TeV, Phys. Rev. Lett. 105 (2010) 022002 [arXiv:1005.3299].

[42] CMS Collaboration, Transverse momentum and pseudorapidity distributions of charged hadrons in pp collisions at $\sqrt{s}=0.9$ and 2.36 TeV, JHEP 1002 (2010) 041 [arXiv:1002.0621].

[43] CMS Collaboration, Jet cross sections and PDF constraints, CMS-PAS-QCD-11-004.

[44] M. Wobisch et al. [fastNLO Collaboration], Theory-Data Comparisons for Jet Measurements in Hadron-Induced Processes, arXiv:1109.1310.

[45] CMS Collaboration, Measurement of Dijet Angular Distributions and Search for Quark Compositeness in pp Collisions at sqrts = 7 TeV, Phys. Rev. Lett. 106 (2011) 201804 [arXiv:1102.2020].

[46] CMS Collaboration, Measurement of the Ratio of the 3-jet to 2-jet Cross Sections in pp Collisions at $\sqrt{s}=7$ TeV, Phys. Lett. B 702 (2011) 336 [arXiv:1106.0647].

[47] CMS Collaboration, First Measurement of Hadronic Event Shapes in pp Collisions at $\sqrt{(s)}=7$ TeV, Phys. Lett. B 699 (2011) 48 [arXiv:1102.0068].

[48] S. Brandt, C. Peyrou, R. Sosnowski and A. Wroblewski, The Principal axis of jets. An Attempt to analyze high-energy collisions as two-body processes, Phys. Lett. 12 (1964) 57.

[49] E. Farhi, A QCD Test for Jets, Phys. Rev. Lett. 39 (1977) 1587, doi:10.1103/PhysRevLett.39.1587.

[50] CMS Collaboration, Dijet Azimuthal Decorrelations in pp Collisions at $\sqrt{s}=7$ TeV, Phys. Rev. Lett. 106 (2011) 122003 [arXiv:1101.5029].

[51] F. Englert and R. Brout, Broken Symmetry and the Mass of Gauge Vector Mesons, Phys. Rev. Lett. 13 (1964) 321, doi:10.1103/PhysRevLett.13.321.

[52] P. W. Higgs, Broken symmetries, massless particles and gauge fields, Phys. Lett. 12 (1964) 132, doi:10.1016/0031-9163(64)91136-9.

[53] P. W. Higgs, Broken Symmetries and the Masses of Gauge Bosons, Phys. Rev. Lett. 13 (1964) 508, doi:10.1103/PhysRevLett.13.508.

[54] G. S. Guralnik, C. R. Hagen and T. W. B. Kibble, Global Conservation Laws and Massless Particles, Phys. Rev. Lett. 13 (1964) 585, doi:10.1103/PhysRevLett.13.585.

[55] P. W. Higgs, Spontaneous Symmetry Breakdown without Massless Bosons, Phys. Rev. 145 (1966) 1156, doi:10.1103/PhysRev.145.1156.

[56] T. W. B. Kibble, Symmetry breaking in nonAbelian gauge theories, Phys. Rev. 155 (1967) 1554, doi:10.1103/PhysRev.155.1554.

[57] R. Barate et al. [LEP Working Group for Higgs boson searches and ALEPH and DELPHI and L3 and OPAL Collaborations], Search for the standard model Higgs boson at LEP, Phys. Lett. B 565 (2003) 61 [hep-ex/0306033], doi:10.1016/S0370-2693(03)00614-2.

[58] Martin Grünewald, Winter 2012 update of the LEP Electroweak Working Group site, http://lepewwg.web.cern.ch/LEPEWWG/plots/winter2012/

[59] CMS Collaboration, Combined results of searches for the standard model Higgs boson in pp collisions at $\sqrt{s}=7 \mathrm{TeV}$, Phys. Lett. B 710 (2012) 26 [arXiv:1202.1488].

[60] CMS Collaboration, Combination of SM, SM4, FP Higgs boson searches, CMS-PAS-HIG-12-008. 
[61] CDF and D0 Collaborations, Evidence for a particle produced in association with weak bosons and decaying to a bottom-antibottom quark pair in Higgs boson searches at the Tevatron, Phys. Rev. Lett. 109 (2012) 071804 [arXiv:1207.6436].

[62] CMS Collaboration, Evidence for a new state decaying into two photons in the search for the standard model Higgs boson in pp collisions, CMS-PAS-SMP-12-014.

[63] CMS Collaboration, Search for the standard model Higgs boson decaying into two photons in pp collisions at $\sqrt{s}=7$ TeV, Phys. Lett. B 710 (2012) 403 [arXiv:1202.1487].

[64] S. Dittmaier et al. [LHC Higgs Cross Section Working Group Collaboration], Handbook of LHC Higgs Cross Sections: 1. Inclusive Observables, arXiv:1101.0593.

[65] CMS Collaboration, Search for a Higgs boson in the decay channel $H \rightarrow Z Z^{*} \rightarrow q \bar{q} l^{-} l^{+}$in $p p$ collisions at $\sqrt{s}=7 \mathrm{TeV}$, JHEP 1204 (2012) 036 [arXiv:1202.1416].

[66] S. Bolognesi, Y. Gao, A. V. Gritsan et al. On the spin and parity of a single-produced resonance at the LHC, Phys. Rev. D 86 (2012) 095031 [arXiv:1208.4018].

[67] CMS Collaboration, CMS at the High-Energy Frontier. Contribution to the Update of the European Strategy for Particle Physics, CMS Note 2012-006, Oct 2012.

[68] L. D. Landau, On the angular momentum of a two-photon system, Dokl. Akad. Nauk Ser. Fiz. 60 (1948) 207.

[69] C. -N. Yang, Selection Rules for the Dematerialization of a Particle Into Two Photons, Phys. Rev. 77 (1950) 242, doi:10.1103/PhysRev.77.242.

[70] Y. Gao, A. V. Gritsan, Z. Guo et al. Spin determination of single-produced resonances at hadron colliders, Phys. Rev. D 81 (2010) 075022 [arXiv:1001.3396].

[71] P. Draper and D. McKeen, Diphotons from Tetraphotons in the Decay of a 125 GeV Higgs at the LHC, Phys. Rev. D 85 (2012) 115023 [arXiv:1204.1061].

[72] HCP'2012 Parallel session - Higgs, 14 November 2012, talk by M. Zanetti (CMS Collaboration), http://kds.kek.jp/conferenceDisplay.py?confId=10808, On the mass and spin-parity of the Higgs boson candidate via its decays to Z boson pairs, arXiv:1212.6639.

[73] A. Leike, The Phenomenology of extra neutral gauge bosons, Phys. Rept. 317 (1999) 143 [arXiv:hep-ph/9805494].

[74] T. G. Rizzo, Z' phenomenology and the LHC, hep-ph/0610104.

[75] A. Ali and W. Wang, Production of the Exotic $1^{--}$Hadrons $\phi(2170), X(4260)$ and $Y_{b}(10890)$ at the LHC and Tevatron via the Drell-Yan Mechanism, Phys. Rev. Lett. 106 (2011) 192001 [arXiv:1103.4587].

[76] L. Randall and R. Sundrum, An Alternative to compactification, Phys. Rev. Lett. 83 (1999) 4690 [hep-th/9906064], doi:10.1103/PhysRevLett.83.4690.

[77] L. Randall and R. Sundrum, A Large mass hierarchy from a small extra dimension, Phys. Rev. Lett. 83 (1999) 3370 [arXiv:hep-ph/9905221].

[78] CMS Collaboration, Search for Resonances in the Dilepton Mass Distribution in pp Collisions at $\sqrt{s}=8 \mathrm{TeV}$, CMS-PAS-EXO-12-015.

[79] CMS Collaboration, Search for heavy narrow dilepton resonances in pp collisions at $\sqrt{s}=7 \mathrm{TeV}$ and $\sqrt{s}=8 \mathrm{TeV}$, arXiv:1212.6175. 
[80] CMS Collaboration, Search for narrow resonances in dilepton mass spectra in pp collisions at $\sqrt{s}=7$ TeV, Phys. Lett. B 714 (2012) 158 [arXiv:1206.1849].

[81] CMS Collaboration, Search for high mass resonances decaying into tau-lepton pairs in pp collisions at $\sqrt{s}=7 \mathrm{TeV}$, Phys. Lett. B 716 (2012) 82 [arXiv:1206.1725].

[82] CMS Collaboration, Search for a narrow spin-2 resonance decaying to a pair of $Z$ vector bosons in the semileptonic final state, arXiv:1209.3807.

[83] CMS Collaboration, Search for $Z^{\prime}$ resonances decaying to t $\bar{t}$ in dilepton+jets final states in $p p$ collisions at $\sqrt{s}=7 \mathrm{TeV}$, arXiv:1211.3338.

[84] CMS Collaboration, Search for leptonic decays of $W^{\prime}$ bosons in pp collisions at $\sqrt{s}=8 \mathrm{TeV}$, CMS-PAS-EXO-12-010.

[85] CMS Collaboration, Search for a $W^{\prime}$ or Techni- $\rho$ Decaying into WZ in pp Collisions at $\sqrt{s}=7 \mathrm{TeV}$, Phys. Rev. Lett. 109 (2012) 141801 [arXiv:1206.0433].

[86] CMS Collaboration, Search for a $W^{\prime}$ boson decaying to a bottom quark and a top quark in pp collisions at $\sqrt{s}=7 \mathrm{TeV}$, Accepted to publication in Phys. Lett. B [arXiv:1208.0956].

[87] CMS Collaboration, Search for Narrow Resonances using the Dijet Mass Spectrum in pp Collisions at $\sqrt{s}=8 \mathrm{TeV}$, CMS-PAS-EXO-12-016.

[88] CMS Collaboration, Search for Resonances in the Dijet Mass Spectrum from 7 TeV pp Collisions at CMS, Phys. Lett. B 704 (2011) 123 [arXiv:1107.4771].

[89] CMS Collaboration, Search for narrow resonances and quantum black holes in inclusive and b-tagged dijet mass spectra from pp collisions at $\sqrt{s}=7 \mathrm{TeV}$, arXiv:1210.2387.

[90] W. -Y. Keung and G. Senjanovic, Majorana Neutrinos And The Production Of The Right-handed Charged Gauge Boson, Phys. Rev. Lett. 50 (1983) 1427, doi:10.1103/PhysRevLett.50.1427.

[91] CMS Collaboration, Search for a heavy neutrino and right-handed $W$ of the left-right symmetric model in pp collisions at $8 \mathrm{TeV}$, CMS-PAS-EXO-12-017.

[92] S. Dimopoulos and G. L. Landsberg, Black holes at the LHC, Phys. Rev. Lett. 87 (2001) 161602 [hep-ph/0106295], doi:10.1103/PhysRevLett.87.161602.

[93] S. B. Giddings and S. D. Thomas, High-energy colliders as black hole factories: The End of short distance physics, Phys. Rev. D 65 (2002) 056010 [hep-ph/0106219], doi:10.1103/PhysRevD.65.056010.

[94] CMS Collaboration, Search for Black Holes in pp Collisions at $\sqrt{s}=8 \mathrm{TeV}$, CMS-PAS-EXO-12-009.

[95] N. Arkani-Hamed, S. Dimopoulos and G. R. Dvali, The Hierarchy problem and new dimensions at a millimeter, Phys. Lett. B 429 (1998) 263 [hep-ph/9803315], doi:10.1016/S0370-2693(98)00466-3.

[96] N. Arkani-Hamed, S. Dimopoulos and G. R. Dvali, Phenomenology, astrophysics and cosmology of theories with submillimeter dimensions and TeV scale quantum gravity, Phys. Rev. D 59 (1999) 086004 [hep-ph/9807344], doi:10.1103/PhysRevD.59.086004.

[97] D. -C. Dai, G. Starkman, D. Stojkovic et al., BlackMax: A black-hole event generator with rotation, recoil, split branes, and brane tension, Phys. Rev. D 77 (2008) 076007 [arXiv:0711.3012].

[98] CMS Collaboration, Search for Microscopic Black Hole Signatures at the Large Hadron Collider, Phys. Lett. B 697 (2011) 434 [arXiv:1012.3375]. 
[99] CMS Collaboration, Search for microscopic black holes in pp collisions at $\sqrt{s}=7$ TeV, JHEP 1204 (2012) 061 [arXiv:1202.6396].

[100] CMS Collaboration, Public CMS Exotica Results web page https://twiki.cern.ch/twiki/bin/view/CMSPublic/PhysicsResultsEXO

[101] CMS Collaboration, Interpretation of Searches for Supersymmetry, CMS-PAS-SUS-11-016.

[102] CMS Collaboration, Public CMS Supersymmetry Results web page https://twiki.cern.ch/twiki/bin/view/CMSPublic/PhysicsResultsSUS

[103] CMS Collaboration, CMS physics technical design report: Addendum on high density QCD with heavy ions, J. Phys. G 34 (2007) 2307, doi:10.1088/0954-3899/34/11/008.

[104] CMS Collaboration, Observation and studies of jet quenching in PbPb collisions at $\sqrt{s_{N N}}=2.76$ TeV, Phys. Rev. C 84 (2011) 024906 [arXiv:1102.1957].

[105] CMS Collaboration, Observation of long-range near-side angular correlations in proton-lead collisions at the LHC, Phys. Lett. B 718 (2013) 795 [arXiv:1210.5482].

[106] CMS Collaboration, Observation of Long-Range Near-Side Angular Correlations in Proton-Proton Collisions at the LHC, JHEP 1009 (2010) 091 [arXiv:1009.4122].

[107] PHOBOS Collaboration, System size dependence of cluster properties from two-particle angular correlations in $C u+C u$ and $A u+A u$ collisions at $\sqrt{s_{N N}}=200 \mathrm{GeV}$, Phys. Rev. C 81 (2010) 024904 [arXiv:0812.1172].

[108] CMS Collaboration, Observation of sequential $\Upsilon$ suppression in PbPb collisions, Phys. Rev. Lett. 109 (2012) 222301 [arXiv:1208.2826].

[109] CMS Collaboration, Indications of suppression of excited $\Upsilon$ states in PbPb collisions at $\sqrt{S_{N N}}=$ 2.76 TeV, Phys. Rev. Lett. 107 (2011) 052302 [arXiv:1105.4894].

[110] CMS Collaboration, Study of high- $p_{T}$ charged particle suppression in PbPb compared to pp collisions at $\sqrt{s_{N N}}=2.76$ TeV, Eur. Phys. J. C 72 (2012) 1945 [arXiv:1202.2554].

[111] CMS Collaboration, Suppression of non-prompt $J / \psi$, prompt $J / \psi$, and $Y(1 S)$ in PbPb collisions at $\sqrt{s_{N N}}=2.76 \mathrm{TeV}$, JHEP 1205 (2012) 063 [arXiv:1201.5069].

[112] CMS Collaboration, Z boson production with the 2011 data in PbPb collisions, CMS-PAS-HIN-12-008.

[113] CMS Collaboration, CMS Physics Results web page https://twiki.cern.ch/twiki/bin/view/CMSPublic/PhysicsResults 